\title{
Divergent trajectories in arithmetic homogeneous spaces of rational rank two
}

\author{
NATTALIE TAMAM \\ Department of Mathematics, Tel Aviv University, Tel Aviv, Israel \\ (e-mail:natalita@post.tau.ac.il)
}

(Received 2 March 2018 and accepted in revised form 21 January 2020)

\begin{abstract}
Let $G$ be a semisimple real algebraic group defined over $\mathbb{Q}, \Gamma$ be an arithmetic subgroup of $G$, and $T$ be a maximal $\mathbb{R}$-split torus. A trajectory in $G / \Gamma$ is divergent if eventually it leaves every compact subset. In some cases there is a finite collection of explicit algebraic data which accounts for the divergence. If this is the case, the divergent trajectory is called obvious. Given a closed cone in $T$, we study the existence of non-obvious divergent trajectories under its action in $G / \Gamma$. We get a sufficient condition for the existence of a non-obvious divergence trajectory in the general case, and a full classification under the assumption that $\operatorname{rank}_{\mathbb{Q}} G=\operatorname{rank}_{\mathbb{R}} G=2$.
\end{abstract}

Key words: group actions, homogeneous dynamics, arithmetic groups, Lie groups representation

2020 Mathematics Subject Classification: 22E40 (Primary); 11J13 (Secondary)

\section{Introduction}

Let $G$ be a semisimple real algebraic group defined over $\mathbb{Q}, \Gamma$ be an arithmetic subgroup of $G$, and $A \subset G$ be a semigroup. The action of $A$ on $G / \Gamma$ induces a flow on $G / \Gamma$. The ergodic theory of these flows is extensively studied and so is the behavior of a generic trajectory. Some sets of exceptional trajectories are related to classical problems in number theory (see [KSS]). A special class of such exceptional trajectories are the divergent trajectories. It was proved by Dani [D] that these trajectories are related to singular systems of linear forms, which are studied in the theory of Diophantine approximation.

A trajectory $A x$ in $G / \Gamma$ is called divergent if the map $a \mapsto a x, a \in A$, is proper. In some cases one can find a simple algebraic reason for the divergence. For example, consider the space of unimodular lattices $\mathrm{SL}_{d}(\mathbb{R}) / \mathrm{SL}_{d}(\mathbb{Z})$ with the action of a one-parameter diagonalizable subgroup $\left\{a_{t}: t \in \mathbb{R}\right\}$. It follows from Mahler's compactness criterion that if one can find a non-zero vector $v \in \mathbb{Z}^{d}$ such that

$$
\left\|a_{t} x \cdot v\right\| \underset{t \rightarrow \infty}{\longrightarrow} 0
$$


then the trajectory $\left\{a_{t} x: t \geq 0\right\}$ is divergent. There are more complicated cases in which one has a sequence of different non-zero vectors $v_{t}$ in $\mathbb{Z}^{d}$ such that $\left\|a_{t} v_{t}\right\| \rightarrow 0$ as $t \rightarrow \infty$. Such trajectories are also divergent but the divergence is not due to one vector $v$.

Given $A$, some natural questions are: Are there divergent trajectories for the action of $A$ on $G / \Gamma$ ? Can one always find a simple reason for the divergence?

For the case discussed in the above example a classification was proved by Dani [D]. He showed that if $d=2$ all divergent trajectories are 'degenerate' and if $d \geq 3$ then there exists a 'non-degenerate' divergent trajectory. What is the difference between these two cases? It seems that the heart of the matter is the rational rank of $G$. In [W1] it was conjectured by Weiss that if $A$ is a diagonal subgroup, then the existence of divergent trajectories and of 'non-obvious' divergent trajectories depends only on the relation between the dimension of $A$ and the rational rank of $G$. See Definitions 1.2 and 1.3 for the definitions of obvious and degenerate divergent trajectories, respectively, and [TW, W1, W2] for results regarding the conjecture.

Assume that $\operatorname{rank}_{\mathbb{Q}} G=\operatorname{rank}_{\mathbb{R}} G=2$. Let $A$ be a two-dimensional closed cone in $G$ (see Definition 1.4 below). Then there are obvious divergent trajectories under the action of $A$. We will show that the existence of non-obvious divergent trajectories under the action of $A$ depends on its intersection with some chambers which are defined using the $\mathbb{Q}$-fundamental weights of $G$. See $\S 1.1$ for the definition of the $\mathbb{Q}$-fundamental weights. These results generalize Corollary 4.7 and Theorem 4.8 in [W1].

We now introduce the terminology and notation we need for stating our main results: Theorem 1.6, which provides a criterion for non-existence of non-obvious divergent trajectories, and Theorem 1.7, which provides a criterion for existence of non-obvious divergent trajectories.

1.1. The main results. Let $G$ be a semisimple real algebraic group defined over $\mathbb{Q}$ and $\Gamma$ be an arithmetic subgroup of $G$ (with respect to the given $\mathbb{Q}$-structure). Denote by $\pi$ the quotient map $G \rightarrow G / \Gamma, g \mapsto g \Gamma$.

Definition 1.1. Let $x \in G / \Gamma$ and $A \subset G$. A trajectory $A x$ is divergent if for any compact subset $K \subset G / \Gamma$ there is a compact $C \subset A$ such that

$$
h \in A \backslash C \Rightarrow h x \notin K .
$$

The easily described divergent trajectories are defined as follows.

Definition 1.2. Let $g \in G$ and let $A \subset G$ be a semigroup. A trajectory $A \pi(g) \subset G / \Gamma$ is called an obvious divergent trajectory if for any unbounded sequence $\left\{a_{k}\right\} \subset A$ there are a subsequence $\left\{a_{k}^{\prime}\right\} \subset\left\{a_{k}\right\}$, a $\mathbb{Q}$-representation $\varrho: G \rightarrow \operatorname{GL}(V)$, and a non-zero $v \in V(\mathbb{Q})$ such that

$$
\varrho\left(a_{k}^{\prime} g\right) v \underset{k \rightarrow+\infty}{\longrightarrow} 0 .
$$

A proof that an obvious divergent trajectory is indeed divergent can be found in [W1].

The notion of degenerate divergent trajectories was defined for one-parameter semigroups of $G$ in [D]. It is similar to the definition of obvious divergent trajectories, but under 
the consideration of a more restricted class of representations. So, the set of degenerate divergent trajectories is a priori smaller than the set of obvious divergent trajectories. The following is a generalization of this definition for any semigroup of $G$.

Definition 1.3. Let $g \in G$ and let $A \subset G$ be a semigroup. A trajectory $A \pi(g) \subset G / \Gamma$ is called a degenerate divergent trajectory if for any unbounded sequence $\left\{a_{k}\right\} \subset A$ there are a subsequence $\left\{a_{k}^{\prime}\right\} \subset\left\{a_{k}\right\}$, a $\mathbb{Q}$-representation $\varrho: G \rightarrow \operatorname{GL}(V)$, and a non-zero $v \in V(\mathbb{Q})$ such that:

(1) $\varrho\left(a_{k}^{\prime} g\right) v \underset{k \rightarrow+\infty}{\longrightarrow} 0$;

(2) $G_{[v]}=\{g \in G: \varrho(g) v$ is a scalar multiple of $v\}$ is a parabolic subgroup of $G$.

Let $\mathfrak{g}$ be the Lie algebra of $G$. Equip $\mathfrak{g}$ with a $\mathbb{Q}$-structure which is $\operatorname{Ad}(\Gamma)$-invariant. Fix some rational basis for $\mathfrak{g}$ and denote its $\mathbb{Z}$-span by $\mathfrak{g}_{\mathbb{Z}}$. Let $S$ be a maximal $\mathbb{Q}$-split torus and let $T$ be a maximal $\mathbb{R}$-split torus which contains $S$. Denote by $\mathfrak{t}$ and $\mathfrak{s}$ the Lie algebras of $T$ and $S$, respectively.

Definition 1.4. A semigroup $A \subset T$ is called a closed cone if there are a connected subgroup $T_{0} \subset T$, finitely many characters $\lambda_{1}, \ldots, \lambda_{k} \in T^{*}$, and non-negative $p_{1}, \ldots, p_{k}$ such that

$$
A=\left\{a \in T_{0}: \forall i=1, \ldots, k, \lambda_{i}(a) \geq p_{i}\right\} .
$$

It is shown in [W1] that obvious divergent trajectories for the action of closed cones are determined by finitely many rational representations and finitely many rational vectors.

Denote by $\Phi_{\mathbb{Q}}$ the set of $\mathbb{Q}$-roots in $\mathfrak{s}^{*}$. Denote by $W\left(\Phi_{\mathbb{Q}}\right)$ the Weyl group associated with $\Phi_{\mathbb{Q}}$, i.e. the group generated by the reflections $\omega_{\lambda}, \lambda \in \Phi_{\mathbb{Q}}$, defined by

$$
\omega_{\lambda}(\chi)=\chi-\langle\chi, \lambda\rangle \lambda
$$

for any characters $\chi$ in $\mathfrak{s}^{*}(\langle\cdot, \cdot\rangle$ will be defined in $\S 2.1)$.

There exists a subset $\Delta_{\mathbb{Q}} \subset \Phi_{\mathbb{Q}}$ such that any $\lambda \in \Phi_{\mathbb{Q}}$ can be expressed uniquely as a linear combination

$$
\lambda=\sum_{\alpha \in \Delta_{\mathbb{Q}}} m_{\alpha}(\lambda) \alpha
$$

in which each $m_{\alpha}(\lambda) \in \mathbb{Z}$ and either all $m_{\alpha}(\lambda) \geq 0$ or all $m_{\alpha}(\lambda) \leq 0$. The set $\Delta_{\mathbb{Q}}$ is called a $\mathbb{Q}$-simple system and each $\alpha \in \Delta_{\mathbb{Q}}$ is called a $\mathbb{Q}$-simple root. For $\lambda_{1}, \lambda_{2} \in \mathfrak{s}^{*}$, we write $\lambda_{1} \geq \lambda_{2}$ if $\lambda_{1}-\lambda_{2}$ can be written as a linear combination of $\mathbb{Q}$-simple roots with non-negative coefficients, and $\lambda_{1}>\lambda_{2}$ if $\lambda_{1} \geq \lambda_{2}$ and $\lambda_{1} \neq \lambda_{2}$.

Fix

$$
\Delta_{\mathbb{Q}}=\left\{\alpha_{1}, \ldots, \alpha_{r}\right\}
$$

Let $\chi_{1}, \ldots, \chi_{r} \in \mathfrak{s}^{*}$ be the $\mathbb{Q}$-fundamental weights of $\mathfrak{g}$, i.e. for any $1 \leq i, j \leq r$,

$$
\left\langle\chi_{i}, \alpha_{j}\right\rangle=\delta_{i, j}
$$

(Kronecker delta).

For $\lambda \in \mathfrak{t}^{*}$, denote by $\left.\lambda\right|_{\mathfrak{s}}$ the restriction of $\lambda$ to $\mathfrak{s}$. 

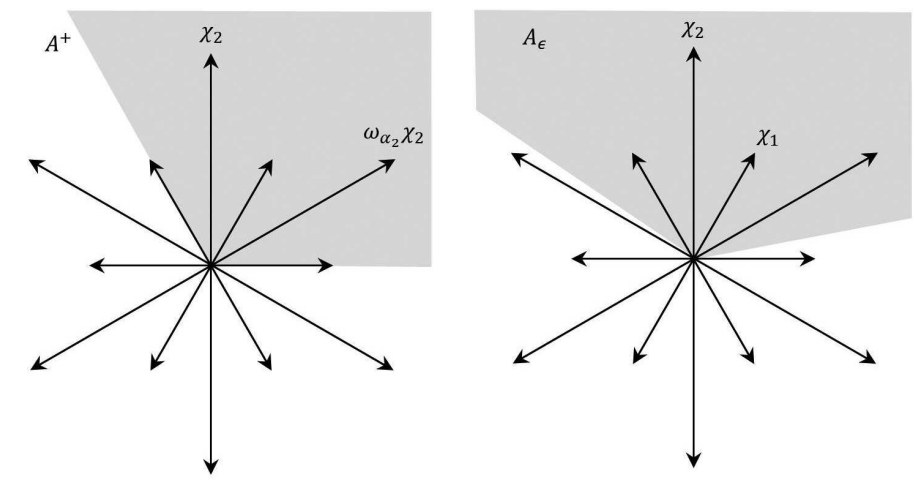

Figure 1. Depiction of $A^{+}$(as defined in Theorem 1.6) and $A_{\epsilon}$ (as defined in Theorem 1.7) for the standard basis of $\mathfrak{g}_{2}$

Remark 1.5. According to [Bor $1, \S 8.5]$, there exists $\mathfrak{t}_{0}$ such that $\mathfrak{t}$ is a direct sum of $\mathfrak{t}_{0}$ and $\mathfrak{s}$. Thus, for any character $\chi \in \mathfrak{s}^{*}$, one can define $\tilde{\chi} \in \mathfrak{t}^{*}$ uniquely by $\left.\tilde{\chi}\right|_{\mathfrak{s}}=\chi$ and $\left.\tilde{\chi}\right|_{\mathfrak{t}_{0}}=0$.

Let $\|\cdot\|$ be a norm on $\mathfrak{t}$.

Our main result provides a necessary condition for the existence of non-obvious divergence trajectories under the action of closed cones in $T$ on $G / \Gamma$.

THEOREM 1.6. Assume that $\operatorname{rank}_{\mathbb{Q}} G=\operatorname{rank}_{\mathbb{R}} G=2$. Let

$$
\begin{aligned}
\mathfrak{a}^{+} & =\left\{t \in \mathfrak{t}: \chi_{1}(t) \geq 0, \omega_{\alpha_{1}}\left(\chi_{1}\right)(t) \geq 0\right\}, \\
A^{+} & =\exp \left(\mathfrak{a}^{+}\right) .
\end{aligned}
$$

Then, for any closed cone $A \supset A^{+}$, there are only degenerate divergent trajectories for the action of $A$ on $G / \Gamma$.

Our second result provides a sufficient condition for the existence of a non-obvious divergence trajectory under the action of closed cones in $T$ on $G / \Gamma$. In particular, it shows that when $\operatorname{rank}_{\mathbb{Q}} G=\operatorname{rank}_{\mathbb{R}} G=2$, any closed cone which does not satisfy the assumption of Theorem 1.6 does not satisfy its conclusion as well. See Figure 1.

THEOREM 1.7. Assume that $\operatorname{rank}_{\mathbb{Q}} G \geq 2$. Let $\epsilon>0$ and let

$$
\begin{aligned}
\mathfrak{a}_{\epsilon} & =\left\{t \in \mathfrak{t}: \tilde{\chi_{1}}(t) \geq \epsilon\|t\|, \tilde{\chi_{2}}(t) \geq \epsilon\|t\|\right\}, \\
A_{\epsilon} & =\exp \left(\mathfrak{a}_{\epsilon}\right) .
\end{aligned}
$$

Then, for any unbounded closed cone $A \subset A_{\epsilon}$, there exists a non-obvious divergent trajectory for the action of $A$ on $G / \Gamma$.

Remark 1.8. A different choice of a simple system or a different indexing of it in (1.3) will result in a different closed cone in Theorem 1.6. In particular, when $\operatorname{rank}_{\mathbb{Q}} G=2$, the two indexing options of the standard basis will result in two different closed cones 

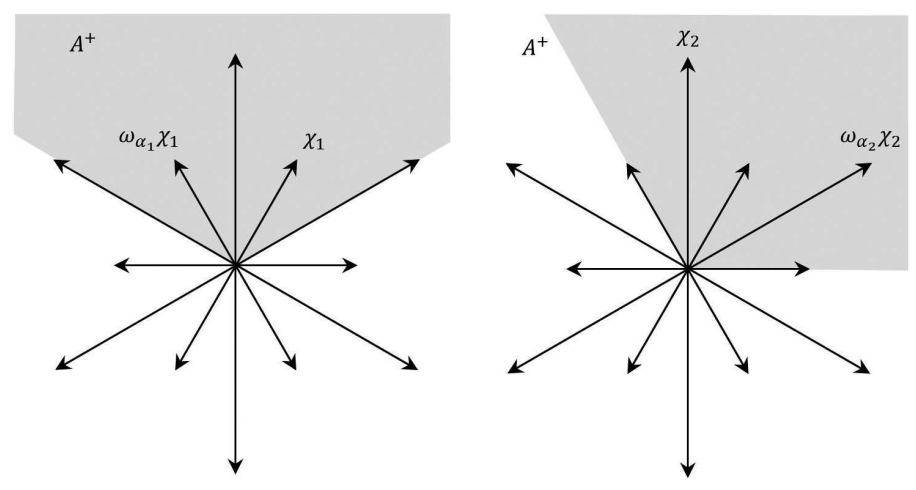

FIGURE 2. Depiction of $A^{+}$(as defined in Theorem 1.6) for the two indexing options of the standard basis of $\mathfrak{g}_{2}$

which are not images of each other by the action of the Weyl group. The Weyl group acts simply transitively on simple systems. Thus, up to the action of the Weyl group, these two examples give all the closed cones which may appear in Theorem 1.6. See Figure 2.

Remark 1.9. It follows from [BT, §2.15] that $G$ is an almost direct product of its $\mathbb{Q}$-almost simple $\mathbb{Q}$-factors. By [BT, §5.11], the relative root system of each $\mathbb{Q}$-almost simple $\mathbb{Q}$-factor is irreducible. Hence, if $\Phi_{\mathbb{Q}}$ is a reducible root system of rational rank two, then up to a finite index $G$ is an almost direct product of $\mathbb{Q}$-subgroups of $G$, each of rational rank one. In that case, Theorem 1.6 follows from [D, Theorem 6.1]. Hence, in the proof of Theorem 1.6 we may assume that $\Phi_{\mathbb{Q}}$ is irreducible.

1.2. Structure. Theorem 1.6 is proved in $\$ 5$. We start by looking at a divergent trajectory under the action of a closed cone. In $\$ 4$ a compactness criterion that can be deduced from [TW, §3] is stated. Using the compactness criterion we can attach to each element in the cone its 'reason for divergence'. The rank assumption in the theorem implies that there are essentially two such reasons. We denote elements in the corresponding sets by $\mathfrak{d}_{1}, \mathfrak{d}_{2}$. Then, up to a compact set, $\mathfrak{d}_{1}$ and $\mathfrak{d}_{2}$ form a cover of $\operatorname{Lie}(A)$. We then use a topological property of $\mathfrak{d}_{1}$ in order to prove the existence of an unbounded connected component in $\mathfrak{d}_{2}$. This topological property is stated in Theorem 4.9. As an important step in the proof of Theorem 4.9, in $\S 3$ we prove Theorem 3.1 regarding the norm of the image of a highest weight vector. In the proof of Theorem 4.9, we also use properties of real representations which are proved in $\S 2.2$ as well as corollaries of the compactness criterion. Once we know that there exist unbounded connected components in $\mathfrak{d}_{1}, \mathfrak{d}_{2}$, we use Proposition 4.8 to find a 'nice' vector in each unbounded component. By using similar arguments if necessary, we then show that there is a finite set of representations and vectors (which were chosen to satisfy the second condition of Definition 1.3) which 'cause' the divergence, proving that the trajectory is a degenerate divergent trajectory.

Theorem 1.7 is proved in $\S 6$. This proof can be read independently of previous sections (apart from notation which appears in $\$ 2$ and $\$ 4$ ). 


\section{Preliminary results}

2.1. Real representations. Denote by $\Phi_{\mathbb{R}}$ the set of $\mathbb{R}$-roots. For $\lambda \in \Phi_{\mathbb{R}}$, denote by $\mathfrak{g}_{\lambda}$ the $\mathbb{R}$-root space for $\lambda$.

Let $\kappa$ be the Killing form on $\mathfrak{g}$ and $\theta$ be the Cartan involution associated with $\kappa$. For $\lambda \in \mathfrak{t}^{*}$, let $t_{\lambda} \in \mathfrak{t}$ be determined by $\lambda(t)=\kappa\left(t_{\lambda}, t\right)$ for all $t \in \mathfrak{t}$. For $\lambda_{1}, \lambda_{2} \in \mathfrak{t}^{*}$, let

$$
\left(\lambda_{1}, \lambda_{2}\right)=\kappa\left(t_{\lambda_{1}}, t_{\lambda_{2}}\right) .
$$

Let $\rho: \mathfrak{g} \rightarrow \mathfrak{g l}(V)$ be an $\mathbb{R}$-representation of $\mathfrak{g}$. For $\lambda \in \mathfrak{t}^{*}$, denote

$$
V_{\rho, \lambda}=\{v \in V: \forall t \in \mathfrak{t}, \rho(t) v=\lambda(t) v\} .
$$

If $V_{\rho, \lambda} \neq\{0\}$, then $\lambda$ is called an $\mathbb{R}$-weight for $\rho$. Denote by $\Phi_{\rho}$ the set of $\mathbb{R}$-weights for $\rho$. For any $\lambda \in \Phi_{\rho}, V_{\rho, \lambda}$ is called the $\mathbb{R}$-weight vector space for $\lambda$, and members of $V_{\rho, \lambda}$ are called $\mathbb{R}$-weight vectors for $\lambda$.

Roots and weights are related by

$$
\rho\left(\mathfrak{g}_{\lambda}\right) V_{\rho, \chi} \subset V_{\rho, \lambda+\chi}, \quad \lambda \in \Phi_{\mathbb{R}}, \chi \in \Phi_{\rho} .
$$

For any $\lambda, \mu \in \mathfrak{t}^{*}$, denote

$$
\langle\lambda, \mu\rangle=2 \frac{(\lambda, \mu)}{(\lambda, \lambda)} .
$$

By [BT, §5], for any $\lambda \in \Phi_{\mathbb{R}}$, real representation $\rho$, and $\mu \in \Phi_{\rho}$,

$$
\langle\lambda, \mu\rangle \text { is an integer. }
$$

The Lie algebra $\mathfrak{s l}(2, \mathbb{R})$ has a basis $H, X, Y$ which satisfies

$$
\operatorname{ad}(H) X=2 X, \quad \operatorname{ad}(H) Y=-2 Y, \quad \operatorname{ad}(X) Y=-H .
$$

LEMMA 2.1. [Bou2, §VIII.1.2] Let $(V, \rho)$ be an irreducible real representation of $\mathfrak{s l}(2, \mathbb{R})$ with dimension $k$. Assume that $V$ is generated by an element $v \in V$ such that $\rho(Y) v=0$ and $\rho(H) v=\lambda v$ for some $\lambda \neq 0$. Then $-\lambda=k-1$.

The following lemma follows from the proof of Proposition 6.52 in [Kn].

LeMmA 2.2. Let $\lambda \in \Phi_{\mathbb{R}}$ and $E \in \mathfrak{g}_{\lambda}$ be non-zero. For some $c>0$. the elements $X=c E$, $Y=\theta X$, and $H=(2 /(\lambda, \lambda)) t_{\lambda}$ satisfy (2.4). In particular, $\mathfrak{v}=\mathbb{R} H \oplus \mathbb{R} X \oplus \mathbb{R} Y$ is a Lie subalgebra of $\mathfrak{g}$ isomorphic to $\mathfrak{s l}(2, \mathbb{R})$.

LEMMA 2.3. Let $(\rho, V)$ be a finite-dimensional real representation of $\mathfrak{g}, \lambda \in \Phi_{\mathbb{R}}, \mu \in \Phi_{\rho}$, $E \in \mathfrak{g}_{\lambda}$, and $v \in V_{\rho, \mu}$. Assume that $\lambda-\mu \notin \Phi_{\rho}$. Then $\langle\lambda, \mu\rangle \leq 0$ and, if

$$
\rho(E)^{l} v=0
$$

for some non-negative $l \leq-\langle\lambda, \mu\rangle$, then $E=0$ or $v=0$.

Proof. According to [Ha, Lemma 10.3], $\lambda-\mu \notin \Phi_{\rho}$ implies that $\langle\lambda, \mu\rangle \leq 0$.

Assume by contradiction that there exists $l \leq\langle\lambda, \mu\rangle$ such that (2.5) is satisfied. Let $X, Y, H, \mathfrak{v}$ be defined as in Lemma 2.2 for $E$. 
Denote

$$
W=\operatorname{span}_{\mathbb{R}}\left\{v, \rho(X) v, \ldots, \rho(X)^{l-1} v\right\} .
$$

It follows from (2.1) and $\lambda-\mu \notin \Phi_{\rho}$ that $\rho(Y) v=0$. Thus, $W$ is invariant under the action of $\mathfrak{v}$. Therefore, $(\rho, W)$ is an irreducible real representation of $\mathfrak{v}$ with dimension $l$. In addition, we have $\rho(H) v=\mu(H) v$ and

$$
\mu(H)=\kappa\left(t_{\mu}, H\right)=\kappa\left(t_{\mu}, \frac{2}{(\lambda, \lambda)} t_{\lambda}\right)=2 \frac{(\mu, \lambda)}{(\lambda, \lambda)}=\langle\lambda, \mu\rangle .
$$

Hence, by Lemma 2.1, we get $-\langle\lambda, \mu\rangle=l-1$, which is a contradiction.

Lemma 2.4. Let $\lambda, \mu \in \Phi_{\mathbb{R}}, X_{\lambda} \in \mathfrak{g}_{\lambda}, X_{2 \lambda} \in \mathfrak{g}_{2 \lambda}$, and $X_{\mu} \in \mathfrak{g}_{\mu}$. Assume that $\lambda-\mu \notin$ $\Phi_{\mathbb{R}} \cup\{0\}$. Then $l=-\langle\lambda, \mu\rangle \geq 0$ and

$$
\sum_{k \leq\left[\frac{l}{2}\right]} \frac{1}{k !} \cdot \frac{1}{(l-2 k) !} \cdot \operatorname{ad}\left(X_{2 \lambda}\right)^{k} \operatorname{ad}\left(X_{\lambda}\right)^{l-2 k} X_{\mu}=0,
$$

implies that $X_{\lambda}=X_{2 \lambda}=0$ or $X_{\mu}=0$.

Proof. It again follows from [Ha, Lemma 10.3] and $\lambda-\mu \notin \Phi_{\rho}$ that $\langle\lambda, \mu\rangle \leq 0$.

Note that if $2 \lambda \notin \Phi_{\mathbb{R}}$, we can deduce the conclusion of the lemma from Lemma 2.3. Hence, we may assume that $2 \lambda \in \Phi_{\mathbb{R}}$. According to (2.3), $\langle 2 \lambda, \mu\rangle$ is an integer. By the linearity of $(\cdot, \cdot)$ and (2.2), we have $\langle\lambda, \mu\rangle=2\langle 2 \lambda, \mu\rangle$. Thus, $l$ is even. If $l=0$, then (2.6) implies that $X_{\mu}=0$. Thus, we may assume that $l>0$. Then, according to [Bou1, §VI.1.3], $l \in\{2,4\}$.

First we will prove that for any $X_{\lambda} \in \mathfrak{g}_{\lambda}, X_{2 \lambda} \in \mathfrak{g}_{2 \lambda}$, and $X_{\mu} \in \mathfrak{g}_{\mu}$,

$$
\operatorname{ad}\left(X_{2 \lambda}\right) X_{\mu}+\operatorname{ad}\left(X_{\lambda}\right)^{2} X_{\mu}=0 \text { implies that } X_{\lambda}=X_{2 \lambda}=0 \text { or } X_{\mu}=0 .
$$

Assume by contradiction that there exist $0 \neq X_{\lambda} \in \mathfrak{g}_{\lambda}$, and $X_{2 \lambda} \in \mathfrak{g}_{2 \lambda}, X_{\mu} \in \mathfrak{g}_{\mu}$ not both zero, such that $\operatorname{ad}\left(X_{2 \lambda}\right) X_{\mu}+\operatorname{ad}\left(X_{\lambda}\right)^{2} X_{\mu}=0$. Then

$$
\operatorname{ad}\left(\theta X_{\mu}\right)\left(\operatorname{ad}\left(X_{2 \lambda}\right) X_{\mu}+\operatorname{ad}\left(X_{\lambda}\right)^{2} X_{\mu}\right)=0 .
$$

By the assumption, $\lambda-\mu \notin \Phi_{\mathbb{R}}$, which implies that $2 \lambda-\mu \notin \Phi_{\mathbb{R}}$. Hence, $\operatorname{ad}\left(\theta X_{\mu}\right)$ commutes with $\operatorname{ad}\left(X_{\lambda}\right)$ and $\operatorname{ad}\left(X_{2 \lambda}\right)$. Therefore, (2.8) implies that

$$
\operatorname{ad}\left(X_{2 \lambda}\right) \operatorname{ad}\left(\theta X_{\mu}\right) X_{\mu}+\operatorname{ad}\left(X_{\lambda}\right)^{2} \operatorname{ad}\left(\theta X_{\mu}\right) X_{\mu}=0 .
$$

By Lemma 2.2, the definition of $t_{\mu}$, and the anti-symmetry of the Lie brackets, we arrive at

$$
\begin{aligned}
0 & =\operatorname{ad}\left(X_{2 \lambda}\right) t_{\mu}+\operatorname{ad}\left(X_{\lambda}\right)^{2} t_{\mu} \\
& =\lambda\left(t_{\mu}\right)\left(2 X_{2 \lambda}+\operatorname{ad}\left(X_{\lambda}\right) X_{\lambda}\right) \\
& =2(\lambda, \mu) X_{2 \lambda} .
\end{aligned}
$$

Thus, $X_{2 \lambda}=0$. Now, according to Lemma 2.3, either $X_{\lambda}=0$ or $X_{\mu}=0$, which is a contradiction. 
Assume that $X_{\lambda} \in \mathfrak{g}_{\lambda}, X_{2 \lambda} \in \mathfrak{g}_{2 \lambda}$, and $X_{\mu} \in \mathfrak{g}_{\mu}$ satisfy (2.6). If $l=2$, then (2.6) implies that

$$
\operatorname{ad}\left(X_{2 \lambda}\right) X_{\mu}+\frac{1}{2} \operatorname{ad}\left(X_{\lambda}\right)^{2} X_{\mu}=0 .
$$

By replacing $X_{\lambda}$ with $\frac{1}{\sqrt{2}} X_{\lambda}$, the conclusion of the lemma follows from (2.7).

If $l=4$, then (2.6) implies that

$$
\frac{1}{2} \cdot \operatorname{ad}\left(X_{2 \lambda}\right)^{2} X_{\mu}+\frac{1}{2} \cdot \operatorname{ad}\left(X_{2 \lambda}\right) \operatorname{ad}\left(X_{\lambda}\right)^{2} X_{\mu}+\frac{1}{4 !} \cdot \operatorname{ad}\left(X_{\lambda}\right)^{4} X_{\mu}=0 .
$$

One can find $c_{1}, c_{2}>0$ such that the left-hand side of (2.10) is equal to

$$
\frac{1}{2}\left(\operatorname{ad}\left(X_{2 \lambda}\right)+c_{1} \operatorname{ad}\left(X_{\lambda}\right)^{2}\right)\left(\operatorname{ad}\left(X_{2 \lambda}\right) X_{\mu}+c_{2} \operatorname{ad}\left(X_{\lambda}\right)^{2} X_{\mu}\right) .
$$

Thus, by replacing $X_{\lambda}$ with $\sqrt{c_{1}} X_{\lambda}$ or $\sqrt{c_{2}} X_{\lambda}$, the conclusion of the lemma follows from (2.7).

2.2. The fundamental weights. Recall that $\Delta_{\mathbb{Q}}$ is a $\mathbb{Q}$-simple system and $\chi_{1}, \ldots, \chi_{r}$ are the corresponding $\mathbb{Q}$-fundamental weights (defined in $\S 1.1$ ).

As in the previous section, $\Phi_{\mathbb{R}}$ is the set of $\mathbb{R}$-roots. According to [Bor1, §21.8], there exists an $\mathbb{R}$-simple system $\Delta_{\mathbb{R}} \subset \Phi_{\mathbb{R}}$ such that the order on $\Phi_{\mathbb{R}}$ defined using this simple system satisfies

$$
\alpha>\left.\beta \Rightarrow \alpha\right|_{\mathfrak{s}} \geq\left.\beta\right|_{\mathfrak{s}}
$$

Denote by $\Phi_{\mathbb{R}}^{+}$the set of positive $\mathbb{R}$-roots, i.e. the roots $\lambda \in \Phi_{\mathbb{R}}$ such that $\lambda>0$.

If the only multiples of a $\mathbb{Q}$-root $\lambda$ in $\Phi_{\mathbb{Q}}$ are $\pm \lambda$, then $\lambda$ is called reduced. If all $\lambda \in \Phi_{\mathbb{Q}}$ are reduced, then $\Phi_{\mathbb{Q}}$ is called reduced.

For any $\alpha \in \Delta_{\mathbb{Q}}$, let

$$
\Phi_{\alpha}=\left\{\beta \in \Phi_{\mathbb{R}}: \Phi_{\mathbb{Q}} \text { is reduced and }\left.\beta\right|_{\mathfrak{s}} \geq \alpha \text {, or } \Phi_{\mathbb{Q}} \text { is non-reduced and }\left.\beta\right|_{\mathfrak{s}} \geq 2 \alpha\right\}
$$

and

$$
\chi_{\alpha}=\sum_{\beta \in \Phi_{\alpha}} \beta .
$$

LeMma 2.5. For any $\alpha \in \Delta_{\mathbb{Q}}, \beta \in \Phi_{\mathbb{R}}^{+}$,

$$
\left\langle\chi_{\alpha}, \beta\right\rangle \begin{cases}\geq 0 & \text { if }\left.\beta\right|_{\mathfrak{s}} \geq \alpha, \\ =0 & \text { otherwise }\end{cases}
$$

Proof. Let $\beta \in \Phi_{\mathbb{R}}^{+}$be such that $\left.\beta\right|_{\mathfrak{s}} \nsucceq \alpha$. Then, equation (1.1) implies that for any $\lambda \in \Phi_{\alpha}$ we have $\omega_{\beta}(\lambda) \in \Phi_{\alpha}$. Hence, $\omega_{\beta}$ leaves $\Phi_{\alpha}$ invariant. Thus, (2.13) implies that $\omega_{\beta}\left(\chi_{\alpha}\right)=$ $\chi_{\alpha}$. If follows from (1.1) that $\left\langle\chi_{\alpha}, \beta\right\rangle=0$, proving the second part of the lemma.

Let $\left.\beta\right|_{\mathfrak{s}} \geq \alpha$. Then, for any $\lambda \in \Phi_{\alpha}$, either $\omega_{\beta}(\lambda) \in \Phi_{\alpha}$ or $\omega_{\beta}(\lambda)<\lambda$. Therefore,

$$
\omega_{\beta}\left(\chi_{\alpha}\right) \leq \chi_{\alpha}
$$

The first part of the lemma now follows from (1.1). 
Remark 2.6. In a similar way to Remark 1.5 , one can deduce from Lemma 2.5 that for any $1 \leq i \leq r, \chi_{\alpha_{i}}$ is a scalar multiple of $\tilde{\chi}_{i}$.

LEMMA 2.7. Assume that $\operatorname{rank}_{\mathbb{Q}} G=2$, and $\Phi_{\mathbb{Q}}$ is irreducible. For any $\omega \in W\left(\Phi_{\mathbb{Q}}\right)$, there exist $a, b$, not both positive, such that $\omega\left(\chi_{1}\right)=a \chi_{1}+b \omega_{\alpha_{1}}\left(\chi_{1}\right)$.

Proof. Let $\omega \in W\left(\Phi_{\mathbb{Q}}\right)$. Since the statement is satisfied for the identity element in $W\left(\Phi_{\mathbb{Q}}\right)$ and for $\omega_{\alpha_{1}}$, we may assume that $\omega$ is neither of them.

We say that a weight is dominant if it is a non-negative linear combination of the $\mathbb{Q}$-fundamental weights.

It is known (see [Hu, §13.2]) that any rational weight is conjugated under the Weyl group to exactly one dominant weight. Since $\chi_{1}$ is a dominant weight, there exist $a_{1}, b_{1}$, not both positive, such that

$$
\omega\left(\chi_{1}\right)=a_{1} \chi_{1}+b_{1} \chi_{2} .
$$

Note that $\left\{\omega_{\alpha_{1}}\left(\chi_{1}\right), \chi_{2}\right\}$ are the fundamental weights with respect to the simple system $\omega_{\alpha_{1}}(\Delta)$. Thus, in a similar way to (2.15), one can get that there exist $a_{2}, b_{2}$, not both positive, such that

$$
\omega\left(\chi_{1}\right)=a_{2} \omega_{\alpha_{1}}\left(\chi_{1}\right)+b_{2} \chi_{2} .
$$

Using (1.4), it can be checked that for some $c>0$,

$$
\chi_{2}=c\left(\omega_{\alpha_{1}}\left(\chi_{1}\right)+\chi_{1}\right) .
$$

It follows from (1.4), (2.15), and (2.16) that

$$
\begin{aligned}
a_{1} & =\left\langle\omega\left(\chi_{1}\right), \alpha_{1}\right\rangle \\
& =-\left\langle\omega\left(\chi_{1}\right),-\alpha_{1}\right\rangle \\
& =-\left\langle\omega\left(\chi_{1}\right), \omega_{\alpha_{1}}\left(\alpha_{1}\right)\right\rangle \\
& =-a_{2} .
\end{aligned}
$$

Hence, $b_{1}, b_{2}$ are not both positive. Without loss of generality, assume that $b_{1} \leq 0$. Then, by (2.15) and (2.17), we arrive at

$$
\omega\left(\chi_{1}\right)=\left(a_{1}+c b_{1}\right) \chi_{1}+c b_{1} \omega_{\alpha_{1}}\left(\chi_{1}\right)
$$

with $c b_{1} \leq 0$.

2.3. Strongly rational representations. Let $\varrho: G \rightarrow \mathrm{GL}(V)$ be an $\mathbb{R}$-representation and $\rho: \mathfrak{g} \rightarrow \mathfrak{g l}(V)$ be its derivative. It will be convenient notationally to refer to $\Phi_{\rho}$ as $\Phi_{\varrho}$ and for any $\lambda \in \Phi_{\rho}$ to $V_{\rho, \lambda}$ as $V_{\varrho, \lambda}$ (in the notation of $\S 2.1$ ). Moreover, elements of $\Phi_{\varrho}$ are called $\mathbb{R}$-weights for $\varrho$, for any $\lambda \in \Phi_{\varrho}, V_{\varrho, \lambda}$ is called the $\mathbb{R}$-weight vector space for $\lambda$, and members of $V_{Q, \lambda}$ are called $\mathbb{R}$-weight vectors for $\lambda$.

An $\mathbb{R}$-weight $\chi$ for $\varrho$ is called an $\mathbb{R}$-highest weight for $\varrho$ if any $\lambda \in \Phi_{\varrho}$ satisfies $\lambda \leq \chi$.

Definition 2.8. A finite-dimensional $\mathbb{R}$-representation $\varrho: G \rightarrow \mathrm{GL}(V)$ is called strongly rational over $\mathbb{R}$ if there is an $\mathbb{R}$-highest weight for $\varrho$ and the $\mathbb{R}$-weight vector space for the 
$\mathbb{R}$-highest weight is of dimension one. It is called strongly rational over $\mathbb{Q}$ if it is strongly rational over $\mathbb{R}$, defined over $\mathbb{Q}$, and the $\mathbb{R}$-weight vector space for the $\mathbb{R}$-highest weight is also defined over $\mathbb{Q}$.

For $\alpha \in \Delta_{\mathbb{Q}}$, let $\mathfrak{u}_{\alpha}=\bigoplus_{\lambda \in \Phi_{\alpha}} \mathfrak{g}_{\lambda}, d_{\alpha}=\operatorname{dim} \mathfrak{u}_{\alpha}$, and $\varrho_{\alpha}: G \rightarrow \operatorname{GL}\left(\bigwedge^{d_{\alpha}} \mathfrak{g}\right)$ be the $d_{\alpha}$ th exterior power of the adjoint representation (see $\$ 2.2$ for the definition of $\Phi_{\alpha}$ and [Sp, Yo, HK] for the definition and properties of the exterior power). For any $\alpha \in \Delta \mathbb{Q}, \varrho_{\alpha}$ is strongly rational over $\mathbb{Q}$ with $\mathbb{R}$-highest weight $\tilde{\chi}_{\alpha}$ and $V_{\varrho_{\alpha}, \tilde{\chi}_{\alpha}}$ is the set of all non-zero vectors of the form

$X_{1} \wedge \cdots \wedge X_{d_{\alpha}}$, where for all $1 \leq j \leq d_{\alpha}$, there exists $\beta \in \Phi_{\alpha}$ such that $X_{j} \in \mathfrak{g}_{\beta}$

(see (2.13) and Remark 2.6). We denote by $\rho_{\alpha}$ the derivative of $\varrho_{\alpha}$.

Lemma 2.9. [Kn, Proposition 2.62] For any reduced root $\lambda \in \Phi_{\mathbb{R}}$, there exists $\omega \in$ $W\left(\Phi_{\mathbb{R}}\right)$ such that $\lambda \in \omega\left(\Delta_{\mathbb{R}}\right)$.

LEMMA 2.10. Let $\alpha \in \Delta_{\mathbb{Q}}, \omega \in W\left(\Phi_{\mathbb{R}}\right), \lambda \in \Phi_{\mathbb{R}}, X_{\lambda} \in \mathfrak{g}_{\lambda}, X_{2 \lambda} \in \mathfrak{g}_{2 \lambda}$, and $v \in V_{\varrho_{\alpha}, \omega\left(\tilde{\chi}_{\alpha}\right)}$. Assume that $\omega\left(\tilde{\chi}_{\alpha}\right)+\lambda \in \Phi_{\varrho_{\alpha}}$. Then $l=-\left\langle\omega\left(\tilde{\chi}_{\alpha}\right), \lambda\right\rangle \geq 1$ and

$$
\sum_{k \leq\left[\frac{l}{2}\right]} \frac{1}{k !} \cdot \frac{1}{(l-2 k) !} \cdot \rho_{\alpha}\left(X_{2 \lambda}\right)^{k} \rho_{\alpha}\left(X_{\lambda}\right)^{l-2 k} v=0
$$

implies that $X_{\lambda}=X_{2 \lambda}=0$ or $v=0$.

Proof. First note that by Lemma 2.9 we may assume that $\omega$ is the identity.

Since $\tilde{\chi}_{\alpha}$ is the highest weight, we may deduce that $-\lambda \in \Phi_{\mathbb{R}}^{+}$and so

$$
\tilde{\chi}_{\alpha}-\lambda \notin \Phi_{\varrho_{\alpha}} .
$$

By [Ha, Lemma 10.3], we have $\left\langle\tilde{\chi}_{\alpha}+\lambda, \lambda\right\rangle \leq 2$, which, using (1.1) and (2.2), implies that $\left\langle\tilde{\chi}_{\alpha}, \lambda\right\rangle \leq-1$. By Lemma 2.5, we then get

$$
-\left.\lambda\right|_{\mathfrak{s}} \geq 2 \alpha
$$

Assume by contradiction that there exist $X_{\lambda} \in \mathfrak{g}_{\lambda}, X_{2 \lambda} \in \mathfrak{g}_{2 \lambda}$, not both zero, and a non-zero $v \in V_{\tilde{\chi}_{\alpha}}$ such that (2.19) is satisfied.

If $X_{2 \lambda}=0$, then by Lemma 2.3, (2.19), and (2.20), we get a contradiction. Thus, we may assume that $X_{2 \lambda} \neq 0$ (and then $\Phi_{\mathbb{Q}}$ is non-reduced).

It follows from (2.18) that for some $Y_{1}, \ldots, Y_{d_{\alpha}} \in \mathfrak{g}$,

$$
v=Y_{1} \wedge \cdots \wedge Y_{d_{\alpha}}
$$

Moreover, for any $1 \leq i \leq d_{\alpha}$, there exist $\beta_{i} \in \Phi_{\alpha}$ such that $Y_{i} \in \mathfrak{g}_{\beta_{i}}$ (there might be $\beta_{i}=\beta_{j}$ for $i \neq j$ ). 
For $1 \leq i \leq d_{\alpha}$, denote $l_{i}=\left\langle\beta_{i}, \lambda\right\rangle$. Then $l=\sum_{i=1}^{d_{\beta}} l_{i}$ implies that

$$
\begin{aligned}
& \sum_{k \leq[l / 2]} \frac{1}{k !} \cdot \frac{1}{(l-2 k) !} \cdot \rho_{\alpha}\left(X_{2 \lambda}\right)^{k} \rho_{\alpha}\left(X_{\lambda}\right)^{l-2 k} v \\
& \quad=\bigwedge_{i=1}^{d_{\alpha}} \sum_{k \leq\left[l_{i} / 2\right]} \frac{1}{k !} \cdot \frac{1}{\left(l_{i}-2 k\right) !} \cdot \rho_{\alpha}\left(X_{2 \lambda}\right)^{k} \rho_{\alpha}\left(X_{\lambda}\right)^{l_{i}-2 k} Y_{i} .
\end{aligned}
$$

According to Corollary 4 in [Sp, §7], $v_{1}, \ldots, v_{n}$ satisfy $v_{1} \wedge \cdots \wedge v_{n}=0$ if and only if they are linearly dependent. Therefore, (2.20), (2.22), and the linear independence of the weight spaces imply that there exist $1 \leq i \leq d_{\alpha}$ and a non-zero $Y \in \mathfrak{g}_{\beta_{i}}$ such that

$$
\sum_{k \leq\left[l_{i} / 2\right]} \frac{1}{k !} \cdot \frac{1}{\left(l_{i}-2 k\right) !} \cdot \rho_{\alpha}\left(X_{2 \lambda}\right)^{k} \rho_{\alpha}\left(X_{\lambda}\right)^{l_{i}-2 k} Y=0 .
$$

Since $\Phi_{\mathbb{Q}}$ is non-reduced, (2.12) implies that $\left.\beta_{i}\right|_{\mathfrak{s}} \geq \alpha$. Since $3 \alpha \notin \Phi_{\mathbb{Q}}$ (a basic property of a root system), using (2.21) we may deduce that $\beta_{i}-\lambda \notin \Phi_{\mathbb{R}}$. Thus, (2.23) is a contradiction to Lemma 2.4.

\section{Highest weight representations}

We preserve the notation of $\S 2$.

Let $\varrho: G \rightarrow \mathrm{GL}(V)$ be an irreducible finite-dimensional $\mathbb{R}$-representation of $G$.

There is a direct sum decomposition

$$
V=\bigoplus_{\lambda \in \Phi_{\varrho, \mathbb{R}}} V_{\varrho, \lambda}
$$

For any $\lambda \in \Phi_{\varrho, \mathbb{R}}$, let $\varphi_{\lambda}: V \rightarrow V_{\varrho, \lambda}$ be the projection associated with (3.1).

The goal of this section is to prove that the norm of the image of a highest weight vector can be estimated by looking at a small subset of its coefficients.

THEOREM 3.1. Let $\varrho: G \rightarrow G L(V)$ be a strongly rational over $\mathbb{R}$ representation with an $\mathbb{R}$-highest weight $\chi$, and $\|\cdot\|$ be a norm on $V$. Assume that either $\Phi_{\mathbb{R}}$ is reduced or $\varrho=\varrho_{\beta}$ for some $\beta \in \Delta_{\mathbb{Q}}$ (see $\left.\S 2.3\right)$. Then there exists $c=c(\varrho,\|\cdot\|)>0$ such that any $g \in G$ and $v \in V_{\varrho, \chi}$ satisfy

$$
\|\varrho(g) v\| \leq c \cdot \max _{\omega \in W\left(\Phi_{\mathbb{R}}\right)}\left\|\varphi_{\omega(\chi)}(\varrho(g) v)\right\| .
$$

For the rest of this section assume that $\varrho$ is strongly rational over $\mathbb{R}$ and either $\Phi_{\mathbb{R}}$ is reduced or $\varrho=\varrho_{\beta}$ for some $\beta \in \Delta_{\mathbb{R}}$.

Denote by $\chi$ the highest $\mathbb{R}$-weight for $\varrho$. Let $\rho: \mathfrak{g} \rightarrow \mathfrak{g l}(V)$ be the derivative of $\varrho$.

Let $\|\cdot\|$ be a norm on $V$.

Proposition 3.2. [Bor1, §14.4] Assume that $\Psi=\left\{\lambda_{1}, \ldots, \lambda_{d}\right\} \subset \Phi_{\mathbb{R}}^{+}$is closed under addition, i.e., if $\lambda, \mu \in \Psi$ and $\lambda+\mu \in \Phi_{\mathbb{R}}$, then $\lambda+\mu \in \Psi$. Denote $\mathfrak{n}_{\Psi}=\bigoplus_{i=1}^{d} \mathfrak{g}_{\lambda_{i}}$, $N_{\Psi}=\exp \mathfrak{n}_{\Psi}$ and, for $1 \leq i \leq d$, denote $N_{i}=\exp \mathfrak{g}_{\lambda_{i}}$ (note that $N_{\Psi}$ does not depend 
on how the elements of $\Psi$ are ordered). Then

$$
N_{\Psi}=N_{1} \cdot N_{2} \cdots N_{d}=\left\{n_{1} \cdot n_{2} \cdots n_{d}: n_{i} \in N_{i}\right\}
$$

Let $\mathfrak{n}=\bigoplus_{\lambda \in \Phi_{\mathbb{R}}^{+}} \mathfrak{g}_{\lambda}, N=\exp (\mathfrak{n})$, and $B=N_{G}(N)$. Then $B$ is an $\mathbb{R}$-Borel subgroup.

LeMmA 3.3. Let $\omega \in W\left(\Phi_{\mathbb{R}}\right), n \in N, \xi=\omega(\chi), \alpha \in \Delta_{\mathbb{R}}$, and $v \in V_{\varrho, \xi}$ be non-zero. Assume that $\varphi_{\omega_{\alpha}(\xi)}(\varrho(n) v)=0$ (see (1.1) for the definition of $\left.\omega_{\alpha}\right)$. Then, for any $k \in \mathbb{N}$,

$$
\varphi \xi+k \alpha(\varrho(n) v)=0 .
$$

Proof. Assume that $\Phi_{\mathbb{R}}^{+}=\left\{\lambda_{1}, \ldots, \lambda_{d}\right\}$. Since $\Phi_{\mathbb{R}}^{+}$is closed under addition, we may use Proposition 3.2 to write

$$
n=\exp \left(X_{1}\right) \exp \left(X_{2}\right) \cdots \exp \left(X_{d}\right), \quad X_{i} \in \mathfrak{g}_{\lambda_{i}} .
$$

By the definition of the exponential map, for any $X \in \mathfrak{g}$ we have

$$
\varrho(\exp (X))=\sum_{k=0}^{\infty} \frac{1}{k !} \rho(X)^{k}
$$

It follows from (2.1) that for any $k_{1}, \ldots, k_{d} \in \mathbb{N}, \rho\left(X_{1}\right)^{k_{1}} \cdots \rho\left(X_{d}\right)^{k_{d}} v$ is an $\mathbb{R}$-weight vector for $\xi+k_{1} \lambda_{1}+\cdots+k_{d} \lambda_{d}$. Hence, for any $\mu \in \Phi_{\varrho}$,

$$
\varphi_{\mu}(\varrho(n) v)=\sum_{\substack{\mu=\xi+k_{1} \lambda_{1}+\cdots+k_{d} \lambda_{d} \\ k_{1}, \ldots, k_{d} \in \mathbb{N}}} \frac{1}{k_{1} ! \cdots k_{d} !} \rho\left(X_{1}\right)^{k_{1}} \cdots \rho\left(X_{d}\right)^{k_{d}} v
$$

Assume that $\alpha=\lambda_{i}, 2 \alpha=\lambda_{j}$ and, without loss of generality, assume that $j<i$. Since $\alpha$ is an $\mathbb{R}$-simple root, (3.4) implies that for any $l \in \mathbb{N}$,

$$
\varphi_{\xi+l \alpha}(\varrho(n) v)=\sum_{k \leq[l / 2]} \frac{1}{k !} \frac{1}{(l-2 k) !} \rho\left(X_{j}\right)^{k} \rho\left(X_{i}\right)^{l-2 k} v
$$

(if $2 \alpha \notin \Phi_{\mathbb{R}}$, then $X_{j}=0$ ).

If $\xi+\alpha \notin \Phi_{\varrho}$, then $\rho\left(X_{i}\right) v=0$, and (3.2) can be deduced from (3.5). Assume otherwise.

If $\Phi_{\mathbb{Q}}$ is non-reduced, then equation (3.5) and Lemma 2.10 imply that $X_{i}=X_{j}=0$.

Assume that $\Phi_{\mathbb{Q}}$ is reduced. Then $X_{j}=0$. The maximality of $\chi$, the assumption $\xi+$ $\alpha \in \Phi_{\varrho}$, and Lemma 2.9 imply that $\xi-\alpha \notin \Phi_{\varrho}$. Then, according to Lemma 2.3, $X_{i}=0$.

In both cases $X_{i}=X_{j}=0$ and so (3.2) can be deduced from (3.5).

According to [Bor1, §11.19], the Weyl group satisfies $W\left(\Phi_{\mathbb{R}}\right)=N_{G}(T) / Z_{G}(T)$. For $\omega \in W\left(\Phi_{\mathbb{R}}\right)$, let $\bar{\omega}$ be a representative of $\omega$ in $N_{G}(T)$. For any $\lambda \in \Phi_{\varrho}$ and $\omega \in W\left(\Phi_{\mathbb{R}}\right)$, we have

$$
\rho(\bar{\omega}) V_{\varrho, \lambda}=V_{\varrho, \omega(\lambda)}
$$


THEOREM 3.4. (Bruhat decomposition) [He, §IX.1] We have

$$
G=\biguplus_{\omega \in W\left(\Phi_{\mathbb{R}}\right)} N \bar{\omega} B,
$$

where $\biguplus$ denotes a disjoint union.

Fix an order $\Phi_{\mathbb{R}}^{+}=\left\{\lambda_{1}, \ldots, \lambda_{d}\right\}$ so that

$$
\text { any } 1 \leq i<j \leq d \text { satisfies } \lambda_{i} \ngtr \lambda_{j} \text {. }
$$

For $\omega \in W\left(\Phi_{\mathbb{R}}\right)$, let

$$
\left\{\lambda \in \Phi_{\mathbb{R}}^{+}: \omega(\chi)+\lambda \in \Phi_{\varrho}\right\}=\left\{\lambda_{1}^{\omega}, \ldots, \lambda_{k(\omega)}^{\omega}\right\},
$$

where the indexation is the one induced from the indexation on $\Phi_{\mathbb{R}}^{+}$, and let

$$
U_{\omega}=\exp \left(\mathfrak{g}_{\lambda_{1}^{\omega}}\right) \cdot \exp \left(\mathfrak{g}_{\lambda_{2}^{\omega}}\right) \cdots \exp \left(\mathfrak{g}_{\lambda_{k(\omega)}^{\omega}}\right) .
$$

PROPOSITION 3.5. Let $\omega \in W\left(\Phi_{\mathbb{R}}\right), n \in U_{\omega}$, and $v \in V_{\varrho, \omega(\chi)}$. If $n$ is not the identity and $v$ is non-zero, then there exists $\omega^{\prime} \in W\left(\Phi_{\mathbb{R}}\right)$ such that $\omega^{\prime}(\chi) \neq \omega(\chi)$ and $\varphi_{\omega^{\prime}(\chi)}(\varrho(n) v) \neq 0$.

Proof. Arguing by contradiction, assume that there exist $\omega \in W\left(\Phi_{\mathbb{R}}\right), 1 \neq n \in U_{\omega}$, and $0 \neq v \in V_{\varrho, \omega(\chi)}$ such that any $\omega^{\prime} \in W\left(\Phi_{\mathbb{R}}\right), \omega^{\prime}(\chi) \neq \omega(\chi)$ satisfies $\varphi_{\omega^{\prime}(\chi)}(\varrho(n) v)=0$.

Denote $\xi=\omega(\chi)$. Let $\lambda \in \Phi_{\mathbb{R}}^{+}$be a reduced $\mathbb{R}$-root which satisfies $\xi+\lambda \in \Phi_{\varrho, \mathbb{R}}$. According to Lemma 2.9, there exists $s_{1} \in W\left(\Phi_{\mathbb{R}}\right)$ so that $\lambda \in s_{1}\left(\Delta_{\mathbb{R}}\right)$. Then $s_{1}(\chi)$ is the highest weight according to the order defined by $s_{1}\left(\Delta_{\mathbb{R}}\right)$. Let $v_{1}=\varrho\left(\bar{s}_{1} \bar{\omega}^{-1}\right) v \in V_{\varrho, s_{1}}(\chi)$ and $g=n \bar{\omega} \bar{s}_{1}^{-1} ;$ then

$$
\varrho(n) v=\varrho(g) v_{1} .
$$

Replace $\Delta_{\mathbb{R}}$ with $s_{1}\left(\Delta_{\mathbb{R}}\right)$. By Theorem 3.4, g= $n_{2} \bar{s}_{2} b$, where $n_{2} \in N, s_{2} \in W\left(\Phi_{\mathbb{R}}\right)$, and $b \in B$ (note that here $\Phi_{\mathbb{R}}^{+}$is defined using $s_{1}\left(\Delta_{\mathbb{R}}\right)$ ). Since $s_{1}(\chi)$ is the highest weight, according to the new order, $\varrho(b) v_{1} \in V_{\varrho, s_{1}}(\chi)$. Then there exists a non-zero vector $v_{2} \in$ $V_{\varrho, s_{2} s_{1}(\chi)}$ such that

$$
\varrho(g) v_{1}=\varrho\left(n_{2}\right) v_{2} \text {. }
$$

It follows from (3.3) and $n_{2} \in N$ that $\varphi_{s_{2} s_{1}(\chi)}\left(\varrho\left(n_{2}\right) v_{2}\right)=v_{2}$. Thus, by (3.8) and (3.9), $\varphi_{s_{2} s_{1}(\chi)}(\varrho(n) v)=v_{2}$. Since $\varphi_{s(\chi)}(\varrho(n) v)=0$ if $s(\chi) \neq \xi$, and $v_{2}$ is non-zero, we can deduce that $s_{2} s_{1}(\chi)=\xi$. Since $\xi+\lambda \in \Phi_{\varrho, \mathbb{R}}$, by Lemma 2.10(i) and the assumption, we have $\varphi_{\omega_{\lambda}(\xi)}\left(\varrho\left(n_{2}\right) v_{2}\right)=0$. Then it follows from Lemma 3.3 and equations (3.8) and (3.9) that $\varphi_{\xi+\lambda}(\varrho(n) v)=\varphi_{\xi}+2 \lambda(\varrho(n) v)=0$. Hence, any positive real root $\lambda$ satisfies

$$
\varphi \xi+\lambda(\varrho(n) v)=0 .
$$

By the definition of $U_{\omega}$,

$$
n=\exp \left(X_{1}\right) \cdots \exp \left(X_{k(\omega)}\right), \quad X_{i} \in \mathfrak{g}_{\lambda_{i}^{\omega}} .
$$

We will now show by induction on $i$ that $X_{i}=0$. Let $1 \leq i \leq k(\omega)$ and assume that $X_{1}=\cdots=X_{i-1}=0$. As in the proof of Lemma 3.3, it follows from (3.3), (3.7), and the induction assumption that

$$
\varphi_{\xi+\lambda_{i}^{\omega}}(\varrho(n) v)=\rho\left(X_{i}\right) v
$$


Since $v \neq 0$, by (3.10) and Lemma 2.3, it follows that $X_{i}=0$. Thus, $n$ is the identity element, which is a contradiction.

Let $k_{1}, k_{2} \in \mathbb{N}, l=\left(l_{1}, \ldots, l_{k_{1}}\right) \in \mathbb{R}_{+}^{k_{1}}$. We say that $f: \mathbb{R}^{k_{1}} \rightarrow \mathbb{R}^{k_{2}}$ is l-homogeneous if for any $x_{1}, \ldots, x_{k_{1}} \in \mathbb{R}, a \geq 0$,

$$
f\left(a^{l_{1}} x_{1}, \ldots, a^{l_{k_{1}}} x_{k_{1}}\right)=a f\left(x_{1}, \ldots, x_{k_{1}}\right) .
$$

Let $\|\cdot\|_{l}$ be the following quasi-norm on $\mathbb{R}^{k_{1}}$ :

$$
\|x\|_{l}=\max _{1 \leq i \leq k_{1}}\left|x_{i}\right|^{1 / l_{i}}, \quad x=\left(x_{1}, \ldots, x_{k_{1}}\right) \in \mathbb{R}^{k_{1}} .
$$

LEMMA 3.6. Let $k_{1}, k_{2} \in \mathbb{N}, l \in \mathbb{R}_{+}^{k_{1}}$, and assume a norm on $\mathbb{R}^{k_{2}}$. Let $f: \mathbb{R}^{k_{1}} \rightarrow \mathbb{R}^{k_{2}}$ be a continuous $l$-homogeneous function. Then there exists $c_{1}>0$ such that for any $x \in \mathbb{R}^{k_{1}}$,

$$
c_{1} \cdot\|f(x)\| \leq\|x\|_{l} .
$$

If $f(x)=0, x \in \mathbb{R}^{k_{1}}$, only when $x=0$, then there exists $c_{2}>0$ such that for any $x \in \mathbb{R}^{k_{1}}$,

$$
\|x\|_{l} \leq c_{2} \cdot\|f(x)\| .
$$

The proof of Lemma 3.6 is left as an exercise for the reader.

LEMmA 3.7. [Kn, Theorem 2.63] The $\mathbb{R}$-Weyl group acts simply transitively on $\mathbb{R}$-simple systems. That is, if $\Delta$ and $\Delta^{\prime}$ are two $\mathbb{R}$-simple systems for $\Phi_{\mathbb{R}}$, then there exists one and only one element $\omega \in W\left(\Phi_{\mathbb{R}}\right)$ such that $\omega(\Delta)=\Delta^{\prime}$.

Proposition 3.8. Let $\omega \in W\left(\Phi_{\mathbb{R}}\right)$. For any $c_{1} \geq 1$, there exists $c_{2} \geq 1$ such that if $n \in$ $U_{\omega}, 0 \neq v \in V_{\varrho, \omega(\chi)}$ satisfy

$$
\max _{s \in W\left(\Phi_{\mathbb{R}}\right)}\left\|\varphi_{s(\chi)}(\varrho(n) v)\right\| \leq c_{1} \cdot\|v\|,
$$

then $\|\varrho(n) v\| \leq c_{2} \cdot\|v\|$.

Proof. Let $\left\{E_{1}, \ldots, E_{k}\right\}$ be a basis for $\mathfrak{n}_{\mathbb{R}}$ which satisfies the following. For any $1 \leq i \leq$ $d$, there exists $1 \leq j(i) \leq k, j(0)=1$, such that $\left\{E_{j(i-1)}, \ldots, E_{j(i)}\right\}$ is a basis for $\mathfrak{g}_{\lambda_{i}}$. Let $e \in V_{\varrho, \omega(\chi)}$ be of norm one. By the definition of strongly rational over $\mathbb{R}$ representations and (3.6), the vector $e$ spans $V_{\varrho, \omega(\chi)}$.

For $1 \leq i \leq d$, denote $l_{i}=\sum_{\alpha \in \Delta_{\mathbb{R}}} m_{\alpha}\left(\lambda_{i}\right)$ and, for $\mu \in \Phi_{\varrho}$, denote

$$
l_{\mu}= \begin{cases}1 & \text { if } \mu \leq \omega(\chi), \\ \sum_{\alpha \in \Delta_{\mathbb{R}}} m_{\alpha}(\mu-\omega(\chi)) & \text { otherwise, }\end{cases}
$$

where for an $\mathbb{R}$-weight $\lambda, m_{\alpha}(\lambda)$ is defined as in (1.2). For $\mu \in \Phi_{\varrho}$, define $f_{\mu}: \mathbb{R}^{k} \rightarrow \mathbb{R}$ by

$$
\begin{aligned}
f_{\mu}\left(x_{1}, \ldots, x_{k}\right)=\| & \varphi_{\mu}\left(\varrho \left(\exp \left(x_{1} E_{1}+\cdots+x_{j(1)} E_{j(1)}\right)\right.\right. \\
& \left.\left.\circ \cdots \circ \exp \left(x_{j(d-1)} E_{j(d-1)}+\cdots+x_{k} E_{k}\right)\right) e\right) \|^{1 / l_{\mu}} .
\end{aligned}
$$


If $\mu \geq \omega(\chi)$, it follows from (3.3) that

$$
\begin{aligned}
f_{\mu}\left(x_{1}, \ldots, x_{k}\right)=\| & \sum \frac{1}{b_{1} ! \cdots b_{m} !} \rho\left(x_{j\left(i_{1}-1\right)} E_{j\left(i_{1}-1\right)}+\cdots+x_{j\left(i_{1}\right)} E_{j\left(i_{1}\right)}\right)^{b_{1}} \\
& \circ \cdots \circ \rho\left(x_{j\left(i_{k}-1\right)} E_{j\left(i_{k}-1\right)}+\cdots+x_{j\left(i_{k}\right)} E_{j\left(i_{m}\right)}\right)^{b_{m}} e \|^{1 / l_{\mu}},
\end{aligned}
$$

where the sum is over all $i_{1}<\cdots<i_{m}$ such that $b_{1} \lambda_{i_{1}}+\cdots+b_{m} \lambda_{i_{m}}=\mu-\omega(\chi)$. Since $\mu<\omega(\chi)$ implies that $f_{\mu}(x)=0$, we may deduce that each $f_{\mu}$ is a continuous $l=\left(l_{1}, \ldots, l_{k}\right)$-homogeneous function for any $\mu \in \Phi_{\varrho, \mathbb{R}}$.

Let

$$
\left\{\mu_{1}, \ldots, \mu_{k_{1}}\right\}=\left\{s(\chi): s \in W\left(\Phi_{\mathbb{R}}\right), s(\chi) \neq \omega(\chi)\right\}
$$

and $f: \mathbb{R}^{k} \rightarrow \mathbb{R}^{k_{1}}$ be defined by $f=\left(f_{\mu_{1}}, \ldots, f_{\mu_{k_{1}}}\right)$. Then $f$ is a continuous $l$-homogeneous function and, by Proposition 3.5, $f(x)=0$ implies that $x=0$. Hence, according to Lemma 3.6, there exists $c_{2}^{\prime}>0$ such that for any $x \in \mathbb{R}^{k}$,

$$
\|x\|_{l} \leq c_{2}^{\prime} \cdot\|f(x)\| .
$$

There exists $a=\left(a_{1}, \ldots, a_{k}\right) \in \mathbb{R}^{k}$ such that

$$
n=\exp \left(a_{1} E_{1}+\cdots+a_{j(1)} E_{j(1)}\right) \cdots \exp \left(a_{j(d-1)} E_{j(d-1)}+\cdots+a_{k} E_{k}\right) .
$$

Since $e$ spans $V_{\varrho, \omega(\chi)}$ and is of norm one, and by the linearity of the representation, for any $\mu \in \Phi_{\varrho}$,

$$
\left\|\varphi_{\mu}(\varrho(n) v)\right\|=\|v\| \cdot\left\|f_{\mu}(a)\right\|^{l_{\mu}} .
$$

Then, by (3.12) and the assumption,

$$
\|a\|_{l} \leq c_{2}^{\prime} \cdot\|f(a)\| \leq c_{2}^{\prime} \cdot \frac{1}{\|v\|} \cdot c_{1}\|v\|=c_{2}^{\prime} \cdot c_{1} .
$$

Assume that $\Phi_{\varrho}=\left\{\mu_{1}, \ldots, \mu_{k_{2}}\right\}$. We may apply Lemma 3.6 to $f^{\prime}=\left(f_{\mu_{1}}, \ldots, f_{\mu_{k_{2}}}\right)$, which is also a continuous $l$-homogeneous function, in order to deduce that there exists $c_{1}^{\prime}>0$ such that for any $\mu \in \Phi_{\varrho}, x \in \mathbb{R}^{k}$,

$$
\left\|f_{\mu}(x)\right\| \leq c_{1}^{\prime} \cdot\|x\|_{l} .
$$

Without loss of generality, assume that for any $u \in V$,

$$
\|u\|=\max _{\mu \in \Phi_{Q, \mathbb{R}}}\left\|\varphi_{\mu}(u)\right\| .
$$

Denote $c_{2}=\max _{\mu \in \Phi_{\varrho}}\left(c_{1}^{\prime} \cdot c_{2}^{\prime} \cdot c_{1}\right)^{l_{\mu}}$. Then, by (3.13), (3.14), (3.15), and (3.16),

$$
\begin{aligned}
\|\varrho(n) v\| & =\max _{\mu \in \Phi_{\varrho, \mathbb{R}}}\left\|\varphi_{\mu}(\varrho(n) v)\right\| \\
& =\|v\| \cdot \max _{\mu \in \Phi_{\varrho}}\left\|f_{\mu}(a)\right\|^{l_{\mu}} \\
& \leq c_{2} \cdot\|v\| .
\end{aligned}
$$

Proof of Theorem 3.1. Denote by $c$ the constant $c_{2}$ which satisfies the conclusion of Proposition 3.8 for $c_{1}=1$. 
Let $s \in W\left(\Phi_{\mathbb{R}}\right)$ satisfy

$$
\left\|\varphi_{s(\chi)}(\varrho(g) v)\right\|=\max _{\omega \in W\left(\Phi_{\mathbb{R}}\right)}\left\|\varphi_{\omega(\chi)}(\varrho(g) v)\right\| .
$$

By Theorem 3.7, there exists $s_{1} \in W\left(\Phi_{\mathbb{R}}\right)$ such that $s_{1}\left(\Delta_{\mathbb{R}}\right)=-s\left(\Delta_{\mathbb{R}}\right)$. According to the order defined using $s_{1}\left(\Delta_{\mathbb{R}}\right)$, the $\mathbb{R}$-highest weight for $\varrho$ is $s_{1}(\chi)$. Moreover, $s(\chi)$ is the $\mathbb{R}$-lowest weight for $\varrho$ according to this order, i.e. every $\mathbb{R}$-weight of $\varrho$ is of the form $s(\chi)+\sum_{\alpha \in \Delta_{\mathbb{R}}} m_{\alpha} \alpha$ with non-negative integers $m_{\alpha}$. Let $v^{\prime}=\varrho\left(\bar{s}_{1}\right) v$. Then $v^{\prime} \in V_{\varrho, s_{1}}(\chi)$ and

$$
\varrho(g) v=\varrho\left(g \bar{s}_{1}^{-1}\right) v^{\prime} .
$$

Hence, by replacing $\Delta_{\mathbb{R}}$ with $s_{1}\left(\Delta_{\mathbb{R}}\right), \chi$ with $s_{1}(\chi), v$ with $v^{\prime}$, and $g$ with $g \bar{s}_{1}^{-1}$, we may assume that

$$
\left\|\varphi_{\xi}(\varrho(g) v)\right\|=\max _{\omega \in W\left(\Phi_{\mathbb{R}}\right)}\left\|\varphi_{\omega(\chi)}(\varrho(g) v)\right\|,
$$

where $\xi$ is the $\mathbb{R}$-lowest weight for $\varrho$.

According to Theorem 3.4, $g=n \bar{\omega} b$, where $n \in N, \omega \in W\left(\Phi_{\mathbb{R}}\right)$, and $b \in B$. Since $\chi$ is the highest weight, there exists $v^{\prime} \in V_{\varrho, \omega(\chi)}$ so that

$$
\varrho(g) v=\varrho(n) v^{\prime} .
$$

Since $\xi$ is the lowest weight, $n \in N$, and $\varphi_{\xi}(\varrho(g) v) \neq 0$, by (3.3) we may deduce that $\omega(\chi)=\xi$.

Order $\Phi_{\mathbb{R}}^{+}=\left\{\mu_{1}, \ldots, \mu_{d}\right\}$ so that

$$
\left\{\mu_{1}, \ldots, \mu_{k(\omega)}\right\}=\left\{\lambda_{1}^{\omega}, \ldots, \lambda_{k(\omega)}^{\omega}\right\} .
$$

Using Proposition 3.2 with $\Psi=\Phi_{\mathbb{R}}^{+}$, we may write

$$
n=\exp \left(X_{1}\right) \cdots \exp \left(X_{d}\right), \quad X_{i} \in \mathfrak{g}_{\mu_{i}} .
$$

For $k(\omega)+1 \leq i \leq d$, we have $\xi+\lambda_{i} \notin \Phi_{\varrho}$. Then, by (3.3), we have $\exp \left(X_{i}\right) v=v$. Let $n^{\prime}=\exp \left(X_{1}\right) \cdots \exp \left(X_{k(\omega)}\right)$; then

$$
\varrho(n) v^{\prime}=\varrho\left(n^{\prime}\right) v^{\prime} .
$$

By (3.17), (3.18), and (3.19), we have

$$
\left\|\varphi_{\xi}\left(\varrho\left(n^{\prime}\right) v^{\prime}\right)\right\|=\max _{\omega \in W\left(\Phi_{\mathbb{R}}\right)}\left\|\varphi_{\omega(\chi)}\left(\varrho\left(n^{\prime}\right) v^{\prime}\right)\right\| .
$$

Since $\varphi_{\xi}\left(\varrho\left(n^{\prime}\right) v^{\prime}\right)=v^{\prime}$, Proposition 3.8 implies that

$$
\left\|\varrho\left(n^{\prime}\right) v^{\prime}\right\| \leq c \cdot\left\|\varphi_{\xi}\left(\varrho\left(n^{\prime}\right) v^{\prime}\right)\right\| .
$$

Now (3.18), (3.19), and (3.20) imply the conclusion of the theorem.

The following example shows that the reals in Theorem 3.1 cannot be replaced with the rationals and hence that the assumption on the real rank of $G$ in the proof of Theorem 1.6 is critical. 
Example 3.9. Let

$$
\underline{1}=\left(\begin{array}{ll}
1 & 0 \\
0 & 1
\end{array}\right), \quad i=\left(\begin{array}{cc}
0 & -1 \\
1 & 0
\end{array}\right), \quad j=\left(\begin{array}{cc}
\sqrt{2} & 0 \\
0 & -\sqrt{2}
\end{array}\right), \quad k=\left(\begin{array}{cc}
0 & \sqrt{2} \\
\sqrt{2} & 0
\end{array}\right) .
$$

One can check by direct computation that $D=\mathbb{Q} \cdot 1+\mathbb{Q} \cdot i+\mathbb{Q} \cdot j+\mathbb{Q} \cdot k$ is a central division algebra over $\mathbb{Q}$. Since $D \otimes \mathbb{Q} \mathbb{R} \cong M(2, \mathbb{R})$, the matrix algebra $M(3, D)$ is naturally imbedded in $M(6, \mathbb{R})$. Denote by $G$ the real algebraic group defined over $\mathbb{Q}$ by

$$
G(\mathbb{Q})=\mathrm{SL}_{3}(D)=\{g \in M(3, D): \operatorname{det}(g)=1\} .
$$

Then $G=\mathrm{SL}_{6}(\mathbb{R})$ and

$$
\mathfrak{g}_{\mathbb{Q}}=\{X \in M(3, D): \operatorname{trace}(X)=0\} .
$$

Then

$$
S=\left\{\operatorname{diag}\left(s_{1}, s_{1}, s_{2}, s_{2}, s_{3}, s_{3}\right): s_{1}, s_{2}, s_{3} \in \mathbb{R}, s_{1} \cdot s_{2} \cdot s_{3}=1\right\}
$$

is a maximal $\mathbb{Q}$-split torus in $G$. Let $\varrho: G \rightarrow \operatorname{GL}\left(\bigwedge^{8} \mathfrak{g}\right)$ be the eighth exterior power of the adjoint representation. Then $\varrho$ is a strongly rational over $\mathbb{Q}$-representation. Denote $\underline{0}=\left(\begin{array}{ll}0 & 0 \\ 0 & 0\end{array}\right)$. There exists an order on the rational simple system so that $12 s_{1}$ is the $\mathbb{Q}$-highest weight and

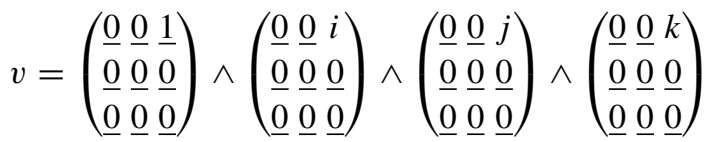

$$
\begin{aligned}
& \wedge\left(\begin{array}{lll}
\underline{0} & \underline{1} & \underline{0} \\
\underline{0} & \underline{0} & \underline{0} \\
\underline{0} & \underline{0} & \underline{0}
\end{array}\right) \wedge\left(\begin{array}{lll}
\underline{0} & i & \underline{0} \\
\underline{0} & \underline{0} & \underline{0} \\
\underline{0} & \underline{0}
\end{array}\right) \wedge\left(\begin{array}{lll}
\underline{0} & j & \underline{0} \\
\underline{0} & \underline{0} & \underline{0} \\
\underline{0} & \underline{0} & \underline{0}
\end{array}\right) \wedge\left(\begin{array}{lll}
\underline{0} & k & \underline{0} \\
\underline{0} & \underline{0} & \underline{0} \\
\underline{0} & \underline{0} & 0
\end{array}\right)
\end{aligned}
$$

is a $\mathbb{Q}$-highest weight vector. Let

$$
g=\left(\begin{array}{llllll}
1 & 0 & 0 & 0 & 0 & 0 \\
0 & 0 & 1 & 0 & 0 & 0 \\
0 & 1 & 0 & 0 & 0 & 0 \\
0 & 0 & 0 & 1 & 0 & 0 \\
0 & 0 & 0 & 0 & 1 & 0 \\
0 & 0 & 0 & 0 & 0 & 1
\end{array}\right) .
$$

Then it can be directly checked that $\varrho(g) v$ is a $\mathbb{Q}$-weight vector for $-6 s_{3}$. Since

$$
\left\{\omega\left(12 s_{1}\right): \omega \in W\left(\Phi_{\mathbb{Q}}\right)\right\}=\left\{12 s_{1}, 12 s_{2}, 12 s_{3}\right\},
$$

we get that

$$
\max _{\omega \in W\left(\Phi_{\mathbb{Q}}\right)}\left\|\varphi_{\omega(\chi)}(\varrho(g) v)\right\|=0
$$

even though $\varrho(g) v$ is a non-zero vector. 


\section{Compactness criterion}

Recall that $\Delta_{\mathbb{Q}}=\left\{\alpha_{1}, \ldots, \alpha_{r}\right\}$ is a $\mathbb{Q}$-simple system for the rational root system $\Phi_{\mathbb{Q}}$.

Let $\mathcal{B}$ be a rational basis for $\mathfrak{g}_{\mathbb{Z}}$ such that $\mathcal{B}_{\beta} \subset \mathfrak{g}_{\beta}$ for any $\beta \in \Phi_{\mathbb{Q}} \cup\{0\}$ and $\mathcal{B}=$ $\biguplus_{\beta \in \Phi_{\mathbb{Q}} \cup\{0\}} \mathcal{B}_{\beta}$.

For $1 \leq i \leq r$, let

$$
\mathfrak{u}_{i}=\bigoplus_{\beta \in \Phi_{\mathbb{Q}}, \beta \geq \alpha_{i}} \mathfrak{g}_{\beta}, \quad P_{i}=N_{G}\left(\mathfrak{u}_{i}\right),
$$

and $\mathcal{B}_{i}=\biguplus_{\beta \geq \alpha_{i}} \mathcal{B}_{\beta}$. Then $P_{1}, \ldots, P_{r}$ are the maximal $\mathbb{Q}$-parabolic subgroups of $G$ containing $B$ (see $\S 3$ for the definition of $B$ ), $\mathfrak{u}_{1}, \ldots, \mathfrak{u}_{r}$ are the Lie algebras of their unipotent radicals, and $\mathcal{B}_{1}, \ldots, \mathcal{B}_{r}$ are bases for $\mathfrak{u}_{1}, \ldots, \mathfrak{u}_{r}$.

Definition 4.1. For a neighborhood $W$ of zero in $\mathfrak{g}, g \in G$, and $1 \leq i \leq r$, we say that $q \in G(\mathbb{Q})$ is $(W, i)$-active for $g$ if

$$
\operatorname{Ad}(g q) \mathcal{B}_{i} \subset \operatorname{Ad}(g) \mathfrak{g}_{\mathbb{Z}} \cap W .
$$

The following useful criterion is similar to the one proved in [TW, Proposition 3.5] and can be deduced from its proof.

Proposition 4.2. (Compactness criterion) There exists a finite subset $C_{0} \subset G(\mathbb{Q})$ which satisfies the following. A subset $A \subset G / \Gamma$ is unbounded if and only if for any neighborhood $W$ of zero in $\mathfrak{g}$ and $g \in G, \pi(g) \in A$, there are $1 \leq i \leq r$, and $q \in \Gamma C_{0}$ which is $(W, i)$-active for $g$.

Remark 4.3. Since $C_{0}$ is a finite subset of $G(\mathbb{Q})$, by changing $\mathcal{B}$ we can assume that $C_{0}$ only contains the identity.

Let $\mathfrak{u}_{0}=\bigoplus_{\beta \in \Phi_{\mathbb{Q}}, \beta>0} \mathfrak{g}_{\beta}$. A subset of $\mathfrak{g}$ is called horospherical if it is contained in a $\mathbb{Q}$-subalgebra conjugate to $\mathfrak{n}_{0}$.

Proposition 4.4. [TW, Proposition 3.3] There exists a neighborhood $W_{0}$ of zero in $\mathfrak{g}$ such that for any $g \in G$, the span of $A d(g) \mathfrak{g}_{\mathbb{Z}} \cap W_{0}$ is horospherical.

Proposition 4.5. [KW, Proposition 3.5] Let $1 \leq i \leq r$. If $\mathfrak{v} \subset \mathfrak{g}$ is conjugate to $\mathfrak{u}_{i}$ and $\mathfrak{v} \subset \mathfrak{n}_{0}$, then $\mathfrak{v}=\mathfrak{u}_{i}$.

PROPOSITION 4.6. There exists a neighborhood $W_{0}$ of zero in $\mathfrak{g}$ such that any neighborhood of zero $W \subset W_{0}$ satisfies the following. Let $A \subset G$ be a connected set and $1 \leq i \leq r$. Assume that for each $g \in A$ there exists $\gamma_{g} \in \Gamma$ which is $(W, i)$-active for $g \in G$. Then, for any $g, h \in A$, we have $\gamma_{g}^{-1} \gamma_{h} \in P_{i}$.

Proof. Let $W_{0}$ be as in Proposition 4.4 and $W \subset W_{0}$.

Let $g \in A$. By the continuity of the adjoint representation, there exists a neighborhood $H$ of $g$ such that for any $h \in H$,

$$
\operatorname{Ad}\left(\gamma_{g}\right) \mathcal{B}_{i} \subset \operatorname{span}\left(\mathfrak{g}_{\mathbb{Z}} \cap \operatorname{Ad}\left(h^{-1}\right) W\right) .
$$


This implies that

$$
\operatorname{Ad}\left(h \gamma_{g}\right) \mathfrak{u}_{i}, \operatorname{Ad}\left(h \gamma_{h}\right) \mathfrak{u}_{i} \subset \operatorname{span}\left(\operatorname{Ad}(h) \mathfrak{g}_{\mathbb{Z}} \cap W\right) .
$$

By Proposition 4.4, the span of $\operatorname{Ad}(h) \mathfrak{g}_{\mathbb{Z}} \cap W$ is contained in a conjugate of $\mathfrak{n}_{0}$. Then Proposition 4.6 implies that $\operatorname{Ad}\left(h \gamma_{g}\right) \mathfrak{u}_{i}=\operatorname{Ad}\left(h \gamma_{h}\right) \mathfrak{u}_{i}$. Therefore, $\gamma_{g}^{-1} \gamma_{h}$ is in the normalizer of $\mathfrak{u}_{i}$, which is $P_{i}$. Since $A$ is connected, the conclusion of the proposition follows.

We use the notation of $\S 2.3$. To simplify some of it, in this section we denote $d_{\alpha_{i}}$ by $d_{i}$ and $\varrho_{\alpha_{i}}$ by $\varrho_{i}$ for any $1 \leq i \leq r$. Note that if $\Phi_{\mathbb{Q}}$ is not reduced, then $\mathfrak{u}_{\alpha_{1}}, \ldots, \mathfrak{u}_{\alpha_{r}}$ are proper subalgebras of $\mathfrak{u}_{1}, \ldots, \mathfrak{u}_{r}$.

For any $1 \leq i \leq r$, let $\Phi_{\alpha_{i}}^{c}$ be the set of all real roots which lie in the span of $\Delta_{\mathbb{Q}} \backslash\left\{\alpha_{i}\right\}$. Then, for any $1 \leq i \leq r, P_{i}$ is the subgroup generated by $Z_{G}(S)$ and $\exp \left(\mathfrak{g}_{\beta}\right)$ for any $\beta \in \Phi_{\alpha_{i}}^{c}$ (see [Bor1, §21.11]). By the definition of $\Phi_{\alpha_{i}}$ and $\Phi_{\alpha_{i}}^{c}, 1 \leq i \leq r$, it is easy to see that for any $\beta \in \Phi_{\alpha_{i}}, \lambda \in \Phi_{\alpha_{i}}^{c}, c_{1}>0$, and $c_{2} \geq 0$ we have $c_{1} \beta+c_{2} \lambda \in \Phi_{\alpha_{i}}$. Hence, for any $1 \leq i \leq r$, the maximality of $P_{i}$ implies that

$$
P_{i}=N_{G}\left(\mathfrak{u}_{\alpha_{i}}\right) .
$$

For any $1 \leq i \leq r$, let $V_{i}=\bigwedge^{d_{i}} \mathfrak{g}$ and

$$
v_{i}=X_{1} \wedge \cdots \wedge X_{d_{i}} \in V_{i}
$$

where

$$
\left\{X_{1}, \ldots, X_{d_{i}}\right\}=\bigcup_{\beta \in \Phi_{\alpha_{i}}} \mathcal{B}_{\beta}=: \mathcal{B}_{i}^{\prime}
$$

is a basis for $\mathfrak{u}_{\alpha_{i}}$ ( $v_{i}$ is uniquely determined up to a sign).

Fix a norm on $\mathfrak{g}$.

The following corollary can be deduced in a similar way to [ $\mathbf{K W}$, Corollary 3.3] using Proposition 4.2, Proposition 4.6, and (4.1).

COROLLARY 4.7. For any $\epsilon>0$, there exists a neighborhood $W_{\epsilon}$ of zero in $\mathfrak{g}$ such that if $W \subset W_{\epsilon}$ is a neighborhood of zero and $\gamma \in \Gamma$ is $(W, i)$-active for $g \in G$, then

$$
\left\|\varrho_{i}(g \gamma) v_{i}\right\|<\epsilon \text {. }
$$

PROPOSITION 4.8. There exists a neighborhood $W_{0}$ of zero in $\mathfrak{g}$ such that any neighborhood of zero $W_{1} \subset W_{0}$ satisfies the following. Let $g \in G, 1 \leq i \leq r, \Psi \subset \Phi_{\varrho_{i}}$, and A be a connected subset of $T$ such that ag is $\left(W_{1}, i\right)$-active for any $a \in A$. If for any neighborhood $W$ of zero in $\mathfrak{g}$ and $\lambda \in \Psi$ there is $a \in A$ such that ag is $(W, i)$-active and $\lambda(a) \geq 1$, then there exists $\gamma \in \Gamma$ such that

$$
\varrho_{i}(g \gamma) v_{i} \in \bigoplus_{\lambda \in \Phi_{\varrho_{i} \backslash \Psi}} V_{\varrho_{i}, \lambda} .
$$

Proof. Let $W_{0}$ be as in Proposition 4.6. For $\lambda \in \Phi_{\varrho_{i}}$, let $\varphi_{\lambda}: V_{i} \rightarrow V_{\varrho_{i}, \lambda}$ be the projection associated with the direct sum decomposition $V_{i}=\bigoplus_{\lambda \in \Phi_{\varrho_{i}}} V_{\varrho_{i}, \lambda}$. 
Let $\lambda \in \Psi$. By Proposition 4.2, Proposition 4.6, Corollary 4.7, and the assumption, there exist $\gamma \in \Gamma$ and a sequence $\left\{a_{j}\right\} \subset A$ such that

$$
\lambda\left(a_{j}\right) \geq 1 \text { and }\left\|\varrho_{i}\left(a_{j} g \gamma\right) v_{i}\right\| \underset{j \rightarrow \infty}{\longrightarrow} 0 .
$$

Since any norm on a finite-dimensional vector space is equivalent to the sup-norm, there exists $c>0$ so that

$$
\left\|\varrho_{i}\left(a_{j} g \gamma\right) v_{i}\right\| \geq c \cdot\left\|\varphi_{\lambda}\left(\varrho_{i}\left(a_{j} g \gamma\right) v_{i}\right)\right\|=c \cdot \lambda\left(a_{j}\right)\left\|\varphi_{\lambda}\left(\varrho_{i}(g \gamma) v_{i}\right)\right\| .
$$

Equations (4.3) and (4.4) prove the conclusion of the proposition.

THEOREM 4.9. Assume that $\operatorname{rank}_{\mathbb{R}} G=\operatorname{rank}_{\mathbb{Q}} G=2$ and $\Phi_{\mathbb{Q}}$ is irreducible. For any $g \in$ $G$, there exists a neighborhood $W_{0}=W_{0}(g)$ of zero in $\mathfrak{g}$ such that any neighborhood of zero $W \subset W_{0}$ satisfies the following. Let $\mathfrak{a}$ be a connected subset of

$$
\mathfrak{a}^{-}=\left\{t \in \mathfrak{t}: \chi_{1}(t) \leq 0, \omega_{\alpha_{1}}\left(\chi_{1}\right)(t) \leq 0\right\}
$$

such that $\exp (a) g$ is $(W, 1)$-active for any $a \in \mathfrak{a}$. Then there exists $\chi \in\left\{\chi_{1}, \omega_{\alpha_{1}} \chi_{1}\right\}$ such that all $a \in \mathfrak{a}$ satisfy $\chi(a)<0$.

Proof. Let the notation be as in the proof of Proposition 4.8.

Assume that $\mathcal{B}=\left\{X_{1}, \ldots, X_{d}\right\}$ and denote the $\mathbb{Z}$-span of

$$
\left\{X_{i_{1}} \wedge \cdots \wedge X_{i_{d_{1}}}: 1 \leq i_{i}<\cdots<i_{d_{1}} \leq d\right\} \subset V_{1}
$$

by $V_{1}(\mathbb{Z})$. Then, $\varrho_{1}(g) V_{1}(\mathbb{Z})$ is a discrete subset. Therefore, there exists $\epsilon_{0}=\epsilon_{0}(g)>0$ such that

$$
\text { for all } v \in \varrho_{1}(g) V_{1}(\mathbb{Z}) \quad\|v\|>\epsilon_{0} .
$$

Let $c$ satisfy the conclusions of Theorem 3.1. Let $W$ satisfy the conclusion of Corollary 4.7 for $\epsilon=\epsilon_{0} / 2 c$. Without loss of generality, assume that $W \subset W_{0}$. Then, according to Corollary 4.7 and Proposition 4.6, there is $\gamma \in \Gamma$ such that for all $a \in \mathfrak{a}$,

$$
\left\|\varrho_{1}(\exp (a) g \gamma) v_{1}\right\|<\epsilon=\frac{\epsilon_{0}}{2 c} .
$$

For any $\lambda \in \Phi_{\varrho_{1}}$, we have

$$
\varphi_{\lambda}\left(\varrho_{1}(\exp (a) g \gamma) v_{1}\right)=\exp (\lambda(a)) \varphi_{\lambda}\left(\varrho_{1}(g \gamma) v_{1}\right) .
$$

Let $s \in W\left(\Phi_{\mathbb{R}}\right)$ satisfy

$$
\left\|\varphi_{s\left(\chi_{1}\right)}\left(\varrho_{1}(g \gamma) v_{1}\right)\right\|=\max _{\omega \in W\left(\Phi_{\mathbb{R}}\right)}\left\|\varphi_{\omega\left(\chi_{1}\right)}\left(\varrho_{1}(g \gamma) v_{1}\right)\right\| .
$$

Then, by Theorem 3.1 and (4.6),

$$
\left\|\varphi_{s\left(\chi_{1}\right)}\left(\varrho_{1}(g \gamma) v_{1}\right)\right\|>\frac{\epsilon_{0}}{c} .
$$

By Lemma 2.7, there exist $b_{1}, b_{2}$, not both positive, such that $s\left(\chi_{1}\right)=b_{1} \chi_{1}+b_{2} \omega_{\alpha_{1}}\left(\chi_{1}\right)$. If $b_{1} \leq 0$ and there exists $a \in \mathfrak{a}$ such that $\omega_{\alpha_{1}} \chi_{1}(a)=0$, the, by (4.5), $s\left(\chi_{1}\right)(a) \geq 0$. Using (4.8), we arrive at

$$
\left\|\varphi_{s\left(\chi_{1}\right)}\left(\varrho_{1}(\exp (a) g \gamma) v_{1}\right)\right\| \geq\left\|\varphi_{s\left(\chi_{1}\right)}\left(\varrho_{1}(g \gamma) v_{1}\right)\right\| .
$$


But then, using (4.7) and (4.9), we obtain a contradiction. Hence, all $a \in \mathfrak{a}$ satisfy $\omega_{\alpha_{1}} \chi_{1}(a)<0$. In a similar way, if $b_{2} \leq 0$, then all $a \in \mathfrak{a}$ satisfy $\chi_{1}(a)<0$.

\section{Proof of Theorem 1.6}

We keep the notation of $\S 4$.

For the proof of Theorem 1.6, we will need the following results.

THEOREM 5.1. (Lebesgue) [Ka, Theorem 4.3] Let the two-dimensional unit cube $\mathcal{I}=$ $[0,1]^{2}$ be covered by a pair of closed sets $X_{1}, X_{2}$. For $i=1,2$, let $F_{i}^{+}$and $F_{i}^{-}$be the facets of $\mathcal{I}$ defined by $x_{i}=1$ and $x_{i}=0$, respectively. Then some connected component of $X_{i}, i \in\{1,2\}$, intersects both the corresponding opposite facets $F_{i}^{+}$and $F_{i}^{-}$.

THEOREM 5.2. (Riemann) [G, §II] Let D denote a simply connected domain in $\mathbb{C}$ that has more than one boundary point. Then there exists an analytic one-to-one mapping of $D$ onto the disk $\{|z|<1\}$. If the boundary of $D$ is a piecewise smooth curve, then the mapping extends in a unique analytic one-to-one way to the closure of $D$.

Replacing $\Delta_{\mathbb{Q}}$ with $-\Delta_{\mathbb{Q}}$, we may assume that

$$
\mathfrak{a}^{+}=\left\{t \in \mathfrak{t}: \chi_{1}(t) \leq 0, \omega_{\alpha_{1}}\left(\chi_{1}\right)(t) \leq 0\right\} .
$$

Recall that $A^{+}=\exp \left(\mathfrak{a}^{+}\right)$. Let $A^{+} \subset A \subset T$ be a closed cone and let $g \in G$. Suppose that $A \pi(g)$ is a divergent trajectory. We need to prove that it is an obvious divergent trajectory.

Let $W_{0}$ satisfy the conclusions of Proposition 4.8 and Theorem 4.9. Let $W_{1}$ be an open neighborhood of zero in $\mathfrak{g}$ so that its closer satisfies $\overline{W_{1}} \subset W_{0}$.

Fix a norm on $\mathfrak{g}$ and denote

$$
\begin{aligned}
& \mathfrak{a}^{+}(r, R)=\left\{a \in \mathfrak{a}^{+}: r \leq\|a\| \leq R\right\}, \\
& \mathfrak{a}^{+}(r)=\left\{a \in \mathfrak{a}^{+}:\|a\| \geq r\right\}, \\
& \mathfrak{a}_{0}^{+}(r)=\left\{a \in \mathfrak{a}^{+}:\|a\|=r\right\} .
\end{aligned}
$$

According to Proposition 4.2 and Remark 4.3, there exists a compact subset $C \subset A$ such that for any $a \in A \backslash C$ there are $1 \leq i \leq r$ and $\gamma \in \Gamma$ which is $\left(W_{1}, i\right)$-active for $a g$. For $i=1,2$, denote

$$
\mathfrak{d}_{i}=\left\{a \in \mathfrak{a}: \exists \gamma \in \Gamma \text { which is }\left(W_{1}, i\right) \text {-active for } \exp (a) g\right\} .
$$

Then there exists $r_{0}>0$ such that $\mathfrak{d}_{1}, \mathfrak{d}_{2}$ is a cover of $\mathfrak{a}^{+}\left(r_{0}\right)$. Moreover, it follows from Definition 4.1 that for $i=1,2$ the set $\mathfrak{d}_{i}$ is open in $\mathfrak{a}=\log (a)$ and satisfies

$$
\overline{\mathfrak{d}}_{i} \subset\left\{a \in \mathfrak{a}: \exists \gamma \in \Gamma \text { which is }\left(W_{0}, i\right) \text {-active for } \exp (a) g\right\} .
$$

We first claim that

for all $r>r_{0} \exists \mathcal{P}$ an unbounded connected component of $\overline{\mathfrak{d}}_{2}$ such that $\mathcal{P} \cap \mathfrak{a}_{0}^{+}(r) \neq \emptyset$.

Assume otherwise. Let $r_{1}>r_{0}$. Since $\mathfrak{d}_{2}$ is an open set and $\mathfrak{a}^{+}\left(r_{1}, r_{1}+1\right)$ is a compact set, there are only finitely many connected components of $\mathfrak{d}_{2}$ which intersect $\mathfrak{a}^{+}\left(r_{1}, r_{1}+1\right)$ 
and the set

$$
S_{1}=\left\{\mathcal{P} \text { is a connected component of } \overline{\mathfrak{d}_{2}}, \mathcal{P} \cap \mathfrak{a}_{0}^{+}\left(r_{1}\right) \neq \emptyset\right\}
$$

is finite. Let $R_{1}>\max \left(\|a\|: \exists \mathcal{P} \in S_{1}\right.$ s.t. $\left.a \in \mathcal{P}\right)$. Then there is no connected component of $\overline{\mathfrak{d}_{2}}$ which intersects both $\mathfrak{a}_{0}\left(r_{1}\right), \mathfrak{a}_{0}\left(R_{1}\right)$.

The boundary of $\mathfrak{a}^{+}$is the union of the following two rays from the origin:

$$
\left\{a \in \mathfrak{a}^{+}: \chi_{1}(a)=0\right\} \text { and }\left\{a \in \mathfrak{a}^{+}: \omega_{\alpha_{1}} \chi_{1}(a)=0\right\} .
$$

Denote

$$
\begin{aligned}
& \mathfrak{l}_{1}(r, R)=\left\{a \in \mathfrak{a}^{+}(r, R): \chi_{1}(a)=0\right\}, \\
& \mathfrak{l}_{2}(r, R)=\left\{a \in \mathfrak{a}^{+}(r, R): \omega_{\alpha_{1}} \chi_{1}(a)=0\right\} .
\end{aligned}
$$

Fix a homeomorphism from $\mathfrak{a}^{+}\left(r_{1}, R_{1}\right)$ to the two-dimensional unit cube $\mathcal{I}$ which sends $\mathfrak{l}_{1}\left(r_{1}, R_{1}\right), \mathfrak{l}_{2}\left(r_{1}, R_{1}\right)$ to $F_{1}^{+}, F_{1}^{-}$and $\mathfrak{a}_{0}\left(r_{1}\right), \mathfrak{a}_{0}\left(R_{1}\right)$ to $F_{2}^{+}, F_{2}^{-}$. Then we may apply Theorem 5.1 with $\overline{\mathfrak{d}_{1}}, \overline{\mathfrak{d}_{2}}$ as the cover of $\mathfrak{a}^{+}\left(r_{1}, R_{1}\right)$ to deduce that there is a connected component of $\overline{\mathfrak{d}_{1}}$ which intersects both $\mathfrak{l}_{1}\left(r_{1}, R_{1}\right), \mathfrak{l}_{2}\left(r_{1}, R_{1}\right)$, which is a contradiction to Theorem 4.9, proving (5.2).

Let $\mathcal{D}_{1}, \mathcal{D}_{2}$ be the sets of all unbounded connected components of $\overline{\mathfrak{d}_{1}}, \overline{\mathfrak{d}_{2}}$, respectively. Let $\mathcal{D}=\mathcal{D}_{1} \cup \mathcal{D}_{2}$. Then by (5.2) $\mathcal{D}$ is not empty.

Recall that for $i=1,2, \varrho_{i}: G \rightarrow \mathrm{GL}\left(V_{i}\right)$ is a strongly rational over $\mathbb{Q}$-representation with a set of $\mathbb{R}$-weights $\Phi_{\varrho_{i}}$, an $\mathbb{R}$-highest weight $\chi_{i}$, and $v_{i}$ non-zero $\mathbb{R}$-weight vectors for $\chi_{i}$. For $\lambda \in \Phi_{\varrho_{i}}$, let $\varphi_{\lambda}: V_{i} \rightarrow V_{\varrho_{i}, \lambda}$ be the projection associated with the direct sum decomposition $V_{i}=\bigoplus_{\lambda \in \Phi_{\varrho_{i}}} V_{\varrho_{i}, \lambda}$.

Let $i=1,2$ and $\mathcal{P} \in \mathcal{D}_{i}$. It follows from (5.1), Proposition 4.2, Proposition 4.8, and our assumption that $A \pi(g)$ diverge that there exists $\gamma_{\mathcal{P}} \in \Gamma$ such that for any unbounded $\left\{a_{k}\right\} \subset A$,

$$
\varrho_{i}\left(\operatorname{ag} \gamma_{\mathcal{P}}\right) v_{i} \rightarrow 0 \text { as } k \rightarrow \infty
$$

Denote $v_{\mathcal{P}}=\varrho_{i}\left(g \gamma_{\mathcal{P}}\right) v_{i}$. Denote by $\Psi_{\mathcal{P}}$ the set of weights $\lambda \in \Phi_{\varrho_{i}}$ such that $\varphi_{\lambda}\left(\varrho_{i}\left(g \gamma_{\mathcal{P}}\right) v_{i}\right) \neq 0$.

Let

$$
\begin{array}{r}
\Upsilon_{i}=\left\{\Psi_{\mathcal{P}}: \mathcal{P} \in \mathcal{D}_{i}\right\}, \quad i=1,2, \\
\text { and } \Upsilon \Upsilon \Upsilon=\Upsilon_{1} \cup \Upsilon_{2} .
\end{array}
$$

Since both $\Phi_{\varrho_{1}}, \Phi_{\varrho_{2}}$ are finite, $\Upsilon_{1}, \Upsilon_{2}$ are also finite.

If for any $a \in \mathfrak{a}$ there exists $\Psi \in \Upsilon$ such that $\lambda(a)<0$ for all $\lambda \in \Psi$, then we are done. Indeed, let $\left\{a_{k}\right\} \subset \mathfrak{a}$ be an unbounded sequence. Since $\Upsilon$ is finite, there are $i=1,2$ and $\Psi \in \Upsilon_{i}$ such that for some subsequence $\left\{a_{k_{\ell}}\right\}, \Psi_{a_{k_{\ell}}}=\Psi$. Then, for $\mathcal{P} \in \mathcal{D}_{i}$ which satisfies $\Psi=\Psi_{\mathcal{P}}$, we have

$$
\left\|\varrho_{i}\left(\exp \left(a_{k_{\ell}}\right)\right) v_{\mathcal{P}}\right\| \leq \max _{\lambda \in \Psi}\left(\exp \left(\lambda\left(a_{k_{\ell}}\right)\right)\right) \cdot\left\|v_{\mathcal{P}}\right\| \rightarrow 0 \quad \text { as } k \rightarrow \infty .
$$

Note that it follows from (4.1) that the second part of Definition 1.3 is satisfied for any $v_{\mathcal{P}}$, $\mathcal{P} \in \mathcal{D}$. 

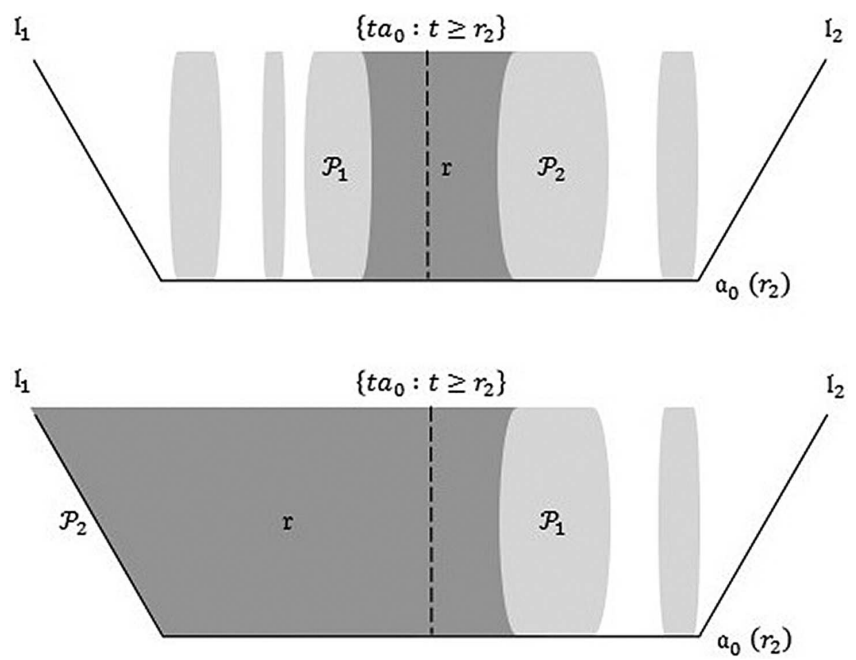

FIGURE 3. Either $\mathfrak{r}$ has elements of $\mathcal{D}$ on both sides, or one of the boundaries of $\mathfrak{r}$ is a boundary of the cone.

Otherwise, there exists $a_{0} \in \mathfrak{a}$ such that $\left\|a_{0}\right\|=1$ and for any $\Psi \in \Upsilon$ there exists $\lambda \in \Psi$ such that $\lambda(a) \geq 0$.

Denote

$$
\begin{aligned}
\mathfrak{a}(r, R) & =\{a \in \mathfrak{a}: r \leq\|a\| \leq R\}, \\
\mathfrak{a}(r) & =\{a \in \mathfrak{a}:\|a\| \geq r\}, \\
\mathfrak{a}_{0}(r) & =\{a \in \mathfrak{a}:\|a\|=r\},
\end{aligned}
$$

and

$$
\epsilon_{0}=\min \left\{\left\|\varphi_{\lambda}\left(v_{\Psi}\right)\right\|: \Psi \in \Upsilon\right\} .
$$

Since $\Phi_{\varrho_{1}}, \Phi_{\varrho_{2}}$ are finite sets, and $g \Gamma$ is a discrete set, $\epsilon_{0}$ is non-zero.

Let $W_{2} \subset W_{0}$ be an open set which satisfies the conclusion of Corollary 4.7 for $0<$ $\epsilon<\epsilon_{0}$. We can repeat the above arguments with $W_{2}$ instead of $W_{0}$ (and maybe get a larger $\left.\epsilon_{0}\right)$. In that case, by Proposition 4.8 , any $\mathcal{P} \subset \mathcal{D}$ satisfies

$$
\mathcal{P} \cap \mathfrak{a}\left(r_{1}\right) \subset\left\{a \in \mathfrak{a}\left(r_{1}\right): \forall \lambda \in \Psi_{\mathcal{P}}, \lambda(a)<0\right\} .
$$

Hence, our choice of $\epsilon$ implies that

$$
\text { for all } \mathcal{P} \in \mathcal{D}, \quad \mathcal{P} \cap\left\{t a_{0}: t \geq r_{1}\right\}=\emptyset .
$$

Since $\mathfrak{a}$ is a closed cone, there exist $\mathfrak{l}_{1}, \mathfrak{l}_{2} \subset \mathfrak{a}$, two disjoint rays from the origin, so that their union is the boundary of $\mathfrak{a}$. Let $\mathcal{P}_{1}, \mathcal{P}_{2} \in \mathcal{D} \cup\left\{\mathfrak{l}_{1}, \mathfrak{l}_{2}\right\}$ be the sets adjacent to the line $\left\{t a_{0}: t \geq r_{1}\right\}$ on the line $\mathfrak{a}_{0}\left(r_{2}\right)$. Note that by (5.2), we may assume that $\mathcal{P}_{1} \in \mathcal{D}$. Let $\mathfrak{r}$ be the maximal closed connected subset of $\mathfrak{a}\left(r_{1}\right)$ such that $\left\{t a_{0}: t \geq r_{1}\right\} \subset \mathfrak{r}$ and $\mathfrak{r}$ does not intersect the interior of $\mathcal{P}_{1} \cup \mathcal{P}_{2}$. See Figure 3 .

Without loss of generality, assume that $\mathcal{P}_{1} \subset \overline{\mathfrak{d}_{2}}$. As before, since $\mathfrak{d}_{2}$ is an open set and $\mathfrak{a}\left(r_{1}, r_{1}+1\right)$ is a compact set, there are only finitely many connected components of $\mathfrak{d}_{1}$ 
which intersect $\mathfrak{a}\left(r_{1}, r_{1}+1\right)$ and the set

$$
S_{2}=\left\{\mathcal{P} \text { is a connected component of } \overline{\mathfrak{d}_{1}}, \mathcal{P} \cap \mathfrak{r} \cap \mathfrak{a}_{0}\left(r_{2}\right) \neq \emptyset\right\} \backslash\left\{\mathcal{P}_{2}\right\}
$$

is finite. Let $R_{2}>\max \left(\|a\|: \exists \mathcal{P} \in S_{2}\right.$ s.t. $\left.a \in \mathcal{P}\right)$. Then there is no connected component of $\overline{\mathfrak{d}_{1}}$ which intersects both $\mathfrak{a}_{0}\left(r_{1}\right), \mathfrak{a}_{0}\left(R_{2}\right)$.

By the definition of $\mathfrak{r}, \mathfrak{r} \cap \mathfrak{a}\left(r_{1}, R_{2}\right)$ is a bounded simply connected domain. Let

$$
\begin{aligned}
& F_{1}^{-}=\mathfrak{r} \cap \mathfrak{a}_{0}\left(r_{1}\right), \quad F_{1}^{+}=\mathfrak{r} \cap \mathfrak{a}_{0}\left(R_{2}\right), \\
& F_{2}^{-}=\mathfrak{r} \cap \overline{\mathcal{P}_{1}} \cap \mathfrak{a}\left(r_{1}, R_{2}\right), \quad F_{2}^{+}=\mathfrak{r} \cap \overline{\mathcal{P}_{2}} \cap \mathfrak{a}\left(r_{1}, R_{2}\right) .
\end{aligned}
$$

Using Theorem 5.2, one can construct a sequence of sets $I_{k} \subset \mathfrak{r} \cap \mathfrak{a}\left(r_{1}, R_{2}\right)$ which satisfy the following.

(1) The boundary of each $I_{k}$ is a disjoint union (up to the end points) of four simple smooth curves $F_{1}^{ \pm}\left(I_{k}\right), F_{2}^{ \pm}\left(I_{k}\right)$ such that for $i=1,2, F_{i}^{+}\left(I_{k}\right)$ and $F_{i}^{-}\left(I_{k}\right)$ are opposite.

(2) Any $\sigma \in\{+,-\}, i \in\{1,2\}$, satisfy $F_{i}^{\sigma}\left(I_{k}\right) \rightarrow F_{i}^{\sigma}$ as $k \rightarrow \infty$.

Moreover, Theorem 5.2 implies that each $I_{k}$ is conformally equivalent to $\mathcal{I}$ with facets $F_{i}^{\sigma}$, $\sigma \in\{+,-\}, i \in\{1,2\}$. Since $\mathfrak{r}$ is covered by $\mathfrak{d}_{1} \cup \mathfrak{d}_{2}$, so is $I_{k}, k \in \mathbb{N}$. Therefore, for any $k \in \mathbb{N}$, we may use Theorem 5.1 with $\overline{\mathfrak{d}_{1}}, \overline{\mathfrak{d}_{2}}$ as a closed cover of $I_{k}$ with facets $F_{1}^{ \pm}, F_{2}^{ \pm}$ to deduce that for some $i \in\{1,2\}$ there exists a connected component $\mathcal{P}_{i, k}$ of $\overline{\mathfrak{d}_{i}}$ which intersects both $F_{i}^{ \pm}\left(I_{k}\right)$. Then there exists $i \in\{1,2\}$ which appears infinitely many times in the sequence $i(1), i(2), \ldots$ Since $\mathfrak{a}\left(r_{1}, R_{2}\right)$ is compact, there exists a non-empty limit set $\mathcal{P}_{i, k} \rightarrow \mathcal{P}_{3}$. Then, by the definition of $R_{2}, \mathcal{P}_{3} \subset \overline{\mathfrak{d}_{2}}$ and intersects $F_{2}^{ \pm}$. Since any connected component of $\overline{\mathfrak{d}_{2}}$ which intersects $\mathcal{P}_{1}$ is $\mathcal{P}_{1}$, we may deduce that $\mathcal{P}_{1}$ intersects $\mathcal{P}_{2}$ in $\mathfrak{r}$, which is a contradiction to the definition of $\mathfrak{r}$.

\section{Proof of Theorem 1.7}

Replacing $\Delta_{\mathbb{Q}}$ with $-\Delta_{\mathbb{Q}}$, we may assume that

$$
\mathfrak{a}_{\epsilon}=\left\{t \in \mathfrak{t}: \chi_{1}^{\prime}(t) \leq-\epsilon\|t\|, \chi_{2}^{\prime}(t) \leq-\epsilon\|t\|\right\}
$$

for some $\epsilon>0$.

We preserve the notation of $\S 4$.

TheOREM 6.1. [W1, Theorem 4.5] Suppose that $G$ is a semisimple $\mathbb{Q}$-algebraic group, $\Gamma=G(\mathbb{Z})$, and $A \subset G$ is a closed cone. Suppose that for $i=1,2$ there are $\mathbb{Q}$-representations $\varsigma_{i}: G \rightarrow G L\left(W_{i}\right)$ and non-zero vectors $w_{i} \in W_{i}(\mathbb{Q})$ such that the following hold.

(1) For any divergent (in $G$ ) sequence $\left\{a_{k}\right\} \subset A$, we have $\varsigma_{i}\left(a_{k}\right) w_{i} \underset{k \rightarrow \infty}{\longrightarrow} 0$ for both $i$.

(2) The groups $Q_{i}=\left\{g \in G: \bar{\varrho}_{i}(g) w_{i} \in \mathbb{R} w_{i}\right\}, i=1,2$, are $\mathbb{Q}$-parabolic subgroups of $G$ and $Q_{1}, Q_{2}$ generate $G$.

Then there is $x \in G$ such that $A \pi(x)$ is divergent, but for any one-parameter semigroup $\{\exp (t a): t \geq 0\} \subset A$, any $\mathbb{Q}$-representation $\varsigma: G \rightarrow G L(W)$, and any non-zero $w \in$ 
$W(\mathbb{Q})$ we have

$$
\varsigma(\exp (\operatorname{ta}) x) w \underset{t \rightarrow+\infty}{\longrightarrow} 0 .
$$

In particular, there are non-obvious divergent trajectories for A.

In the notation of $\S 4$, let $w_{i}=v_{i}$ and $\varsigma_{i}=\varrho_{i}$ for any $i=1,2$. In order to prove Theorem 1.7, it is enough to prove that $v_{1}, v_{2}, \varrho_{1}$, and $\varrho_{2}$ satisfy conditions (1) and (2) of Theorem 6.1 for any $A \subset A_{\epsilon}$.

Let $\left\{\exp \left(a_{k}\right)\right\} \subset A$ be a divergent sequence. Then $\left\|a_{k}\right\| \rightarrow \infty$ as $k \rightarrow \infty$. Without loss of generality, we may assume that the norm defined on $t$ is the sup-norm defined using a basis which contains only $\mathbb{Q}$-weight vectors. Thus, using (2.18) and (4.5) for $i=1,2$, we have

$$
\left\|\varrho_{i}\left(\exp \left(a_{k}\right)\right) v_{i}\right\|=e^{\tilde{\chi}_{i}\left(a_{k}\right)}\left\|v_{i}\right\|<e^{-\epsilon\left\|a_{k}\right\|}\left\|v_{i}\right\| \underset{k \rightarrow \infty}{\longrightarrow} 0
$$

and hence (1) is satisfied.

Since $P_{1}, P_{2}$ are maximal $\mathbb{Q}$-parabolic subgroups of $G$, according to (4.1) in the notation of Theorem 6.1 we have $Q_{i}=P_{i, \mathbb{Q}}$ for $i=1,2$. The maximality of $P_{1}, P_{2}$ implies that condition (2) is also satisfied.

Acknowledgements. I would like to thank my advisor Barak Weiss for his guidance, support, and encouragement as well as many helpful discussions. This research was supported by the Ministry of Science, Technology and Space, Israel, by the European Research Council (ERC) starter grant DLGAPS 279893, and by the Israel Science Foundation (ISF) grant 2095/15.

\section{REFERENCES}

[Bor1] A. Borel. Linear Algebraic Groups (Graduate texts in Mathematics, 126), 2nd enlarged edn. Springer, New York 1991.

[Bou1] N. Bourbaki. Lie Groups and Lie Algebras. Springer, Berlin, 2002, Chs. 4-6.

[Bou2] N. Bourbaki. Lie Groups and Lie Algebras. Springer, Berlin, 2005, Chs. 7-9.

[BT] A. Borel and J. Tits. Groupes réductifs. Publ. Math. Inst. Hautes Études Sci. 27 (1965), 55-150.

[D] G. Dani. Divergent trajectories of flows on homogeneous spaces and diophantine approximation. J. Reine Angew. Math. 359 (1985), 55-89.

[G] G. M. Goluzin. Geometric Theory of Functions of a Complex Variable. American Mathematical Society, Providence, RI, 1969.

[Ha] B. C. Hall. Lie Groups, Lie Algebras, and Representations: An Elementary Introduction (Graduate Texts in Mathematics, 222), 2nd edn. Springer, New York, 2015.

[He] S. Helgason. Differential Geometry, Lie Groups and Symmetric Spaces. Academic Press, New York, 1978.

[HK] K. Hoffman and R. Kunze. Linear Algebra, 2nd edn. Prentice-Hall, Englewood Cliffs, NJ, 1971.

[Hu] J. E. Humphreys. Linear Algebraic Groups (Graduate Texts in Mathematics, 21). Springer, New York, 1975.

[Ka] R. N. Karasev. Covering dimension using toric varieties. Topology Appl. 177 (2014), 59-65.

[Kn] A. W. Knapp. Lie Groups Beyond an Introduction, 2nd edn. Birkhäuser, Boston, MA, 1996.

[KSS] D. Kleinbock, N. A. Shah and A. N. Starkov. Dynamics of subgroup actions on homogeneous spaces of Lie groups and applications to number theory. Handbook of Dynamical Systems. Vol. 1A. North-Holland, Amsterdam, 2002, pp. 813-930.

[KW] D. Kleinbock and B. Weiss. Modified Schmidt games and a conjecture of Margulis. J. Mod. Dyn. 7 (2013), 429-460. 
Divergent trajectories in arithmetic homogeneous spaces of rational rank two 3141

[Sp] M. Spivak. A Comprehensive Introduction to Differential Geometry. Vol. I, 2nd edn. Publish or Perish, Wilmington, DE, 1979.

[TW] G. Tomanov and B. Weiss. Closed orbits for actions of maximal tori on homogeneous spaces. Duke Math. J. 119(2) (2003), 367-392.

[W1] B. Weiss. Divergent trajectories on noncompact parameter spaces. Geom. Funct. Anal. 14(1) (2004), 94-149.

[W2] B. Weiss. Divergent trajectories and $\mathbb{Q}$-rank. Israel J. Math. 152 (2006), 221-227.

[Yo] T. Yokonuma. Tensor Spaces and Exterior Algebra. American Mathematical Society, Providence, RI, 1992. 